\title{
Dynamic Conic Hedging for Competitiveness
}

Dilip B. Madan

Robert H. Smith School of Business

University of Maryland

email: dbm@rhsmith.umd.edu

\author{
Martijn Pistorius \\ Department of Mathematics \\ Imperial College \\ email: m.pistorius@imperial.ac.uk
}

February 25, 2016 


\title{
Dynamic Conic Hedging for Competitiveness
}

\begin{abstract}
The paper provides a new hedging methodology permitting systematic hedging choices with wide applications. Dynamic concave bid price, and convex ask price functionals from the recent literature are employed to construct new hedging strategies termed dynamic conic hedging. The primary focus of these strategies is to adopt positions maximizing a nonlinear conditional expectation expressed recursively as a concave current bid price for the one step ahead risk held or minimizing the convex current ask price for the risk promised. Risk management and hedging then have a new market value enhancing perspective different from the classical forms of risk mitigation, local variance minimization, or even expected utility maximization.

Key Words: Static and Semi Static Hedging, Nonlinear Expectation, Variance Gamma Model, Distorted Expectation.

JEL Classification: G10, G11 and G13.

\section{Introduction}

Recent literature has introduced nonlinear dynamic valuation operators from the perspective of conservative valuation methodologies connected with the theory of coherent risk measures. From the static perspective we may cite Carr, Geman and Madan (2001), Föllmer and Schied (2004), Staum (2004), Barrieu and El Karoui (2005). Dynamic generalizations have been developed for a discrete time context in Cheridito, Delbaen and Kupper (2006), Jobert and Rogers (2005), Detlefsen and Scandolo (2005), Föllmer and Penner (2006), Madan and Schoutens (2012) and Madan (2014a). Continuous time formulations may be found in BionNadal (2008,2009), Eberlein, Madan, Pistorius, Schoutens and Yor (2012) and Eberlein, Madan, Pistorius and Yor (2014). When the set of acceptable risks at any point of time is closed under the scaling of positions by a positive multiple it forms a cone and we refer to the resulting economy as a conic one. For conic economies the bid and ask prices scale with the size of the trade. We restrict our attention in this paper to such conic economies.

Given a dynamic nonlinear conditional expectation operator constructed respectively as either a conic valuation functional for the bid price or for the ask price, one may seek to design hedging strategies to maximize the concave bid price for positions held or minimize the convex ask price for positions promised. For any set of chosen hedging instruments such optimization problems are stochastic control problems related to nonlinear valuation functionals. The associated continuous time stochastic control theory is at the time of writing not yet available. However we may formulate and illustrate a discrete time backward recursion that solves for the hedges and their related optimized bid and ask prices. One is then able to observe the competitive benefits of different hedging instruments and hedging
\end{abstract}


strategies in terms of their ability to deliver higher bid and simultaneously lower ask prices. The objective of this paper is to develop in discrete time this new perspective on hedging. This new approach seeks to maximize the current bid price for the remaining cash flows not yet determined, as a concave functional of the risk held. Alternatively one may minimize the current ask price, for the same remaining cash flows, as a convex functional of the risk promised. The methods developed are illustrated by hedging classical derivatives using the new procedure.

Madan, Pistorius and Stadje (2015a, 2015b) develop limits of discrete time valuation operators that on a careful choice of the limiting process converge to particular valuation operators selected for the limit that employ measure distortions. It is not clear at this point how the interaction with choosing controls will influence the limiting operator that one is converging to. There is a developing literature on the control of BSDE's and we cite for example Oksendahl and Sulem (2009) but in this context one works with drivers for the BSDE specified abstractly. We work here with explicit drivers in discrete time and their continuous time limit is unclear in the presence of controls. Alternatively, one may work explicitly with the limiting drivers identified in Madan, Pistorius and Stadje (2015b), introduce a control context and attempt to implement the methods of Oksendahl and Sulem (2009). We leave for future research an investigation of such an approach.

A close relative to dynamic conic hedging is the standard and semistatic hedging of options as developed in Carr, Ellis and Gupta (1998), Carr (2011) and Carr and Wu (2013). In static and semi-static hedging positions are sought in short maturity options with a view to replicating the value function, of path-dependent claims or longer dated options, taken at the shorter maturity. Under dynamic conic hedging positions are similarly sought in similar assets, but with no requirement about replication. Hence dynamic conic hedging procedures may be employed to hedge complex functions of multiple market indices that have liquid short maturity options trading. In general replication fails in such contexts. Dynamic conic hedging could also be applied in hedging the risks of some insurance liabilities. The liabilities, for example, could be portfolios of variable annuities or guaranteed minimum withdrawal benefit contracts. As such they have complex dependencies of their values on multiple market indices. Such applications would follow on from related work in this direction using static or semi static hedging (Kolkiewicz and Liu (2012), Phillips (2013), Marshall, Hardy and Saunders (2014)).

In contrast to the new perspective on hedging proposed here one may observe that traditionally hedging a risk or liability has been viewed as eliminating the underlying exposure. This is certainly the motivation behind the use of forward contracts to hedge the risk of price movements. Once hedged one is no longer concerned with the risk. Option pricing theory as developed by Black and Scholes (1973) and Merton (1973) showed how to apply these ideas to replicate option liabilities and then to price the option at this replication cost. However, the possibility of replication requires strong assumptions about the stochastic motion of the stock price. Under certain conditions like geometric Brownian motion or its binomial ap- 
proximation one may eliminate the risk and construct what is a perfect hedge. Markets with this property are referred to as complete markets and there are numerous examples of such (see for example Cox, Ross and Rubinstein (1979), Cvitanić and Zapatero (2004)). Leaving aside these complete market examples, one acknowledges that perfect replication may not be possible in actual markets. This places the task of hedging in the context of incomplete markets (Staum (2008)). Nonetheless, the basic intuition to be captured from the Black, Merton and Scholes theoretical advance is the conjecture that dynamic hedging must be helpful in partially reducing the risk of a given derivative contract and thereby enhancing its market value.

This intuition has given rise to numerous papers devoted to hedging in the context of either partial hedging or incomplete markets. Partial hedging is studied, for example, in Föllmer and Leukert (2000), Cvitanić (2000) and Sekine (2004). With regard to incomplete markets we have works on variance minimization, its time inconsistency, and related modifications to local variance minimization (Bertsimas, Kogan and Lo (2001), Heath, Platen and Schweizer (2001), Basak and Chabakauri (2012)). But variance minimization, locally or globally, is burdened by its unrealistic symmetric treatment of gains and losses. Alternatives associated with semi-variance or just the down side variance have been considered and developed (see for example Chen, Lee and Shrestha (2001)). These methods ignore an assessment of the risk beyond the first two moments and are suited for example in situations where the risk is well captured by a Gaussian distribution. Such Gaussian hypotheses are questionable when we consider the post hedge residuals of complex derivatives with a variety of embedded optionalities. We note in this regard that the risk neutral distributions observed in markets are highly non-Gaussian (Christoffersen, Elkhami Feunou and Jacobs (2010)) and reflect varying degrees of skewness and kurtosis for even just a position in the stock, let alone the addition of optionalities. Furthermore, such observed risk neutral departures from Gaussian distributions are critical to the market valuation perspectives we seek to develop here. The departures are generally less pronounced in their physical counterparts or in the possibly subjective beliefs of particular investors.

Yet another approach, respecting gains and accounting for the full distribution of the risk, is to maximize the expected concave utility of the final cash flow or the consumption stream over time. Here losses are shunned with a high associated marginal utility and gains are recognized but discounted by a low marginal utility. The literature on hedging with a view to maximizing expected utility is extensive. Via the concept of certainly equivalent levels of utility are converted back to dollar-values and this gives rise to the theory of indifference pricing (see for example Musiela and Zariphopoulou (2004), Carmona (2009)). Expected utility maximization is embedded in the perspective of an individual investor's rational behavior when faced with uncertainty. The probability measure under which expected utility is evaluated is thereby personalized and may be called into question when employed in the context of representative wealth management. Similar remarks apply to risk attitudes. The objective of expected utility maximization is divorced from, and not cognizant of a financial 
market regularly valuing human and economic activities in open and active markets. Actions that raise expected utility but simultaneously lower market value may not be rational from a market valuation perspective. Other related approaches modeling investor views include robust methods and we cite Hansen and Sargent (2001, 2007), Maenhout (2004), Iyengar and Ma (2010), and Shen, Pelsser and Schotman (2013).

The conservative market valuations considered here provide us with a new perspective on the mathematical design of objectives that could be employed in the study of a variety of corporate and investor decision problems. For example one may design portfolios with a view to maximizing a conservative market value or formulate option positions or dynamic rebalancing strategies with such objectives. Some of these issues are addressed in related papers, for example (Madan and Sharaiha (2014)). We focus here on the dynamic hedging of option positions. The contribution of this paper is to develop conservative value maximizing hedge designs for discrete time incomplete market contexts and to illustrate their implementation for a variety of options and structured products. The methods and procedures developed could be applied to far more complicated situations including the management of insurance liabilities.

We therefore turn our attention to the bid and ask prices of two price economies (Cherny and Madan (2010)), now constructed dynamically (Madan and Schoutens (2012), Eberlein, Madan, Pistorius, Schoutens and Yor (2012), Eberlein, Madan, Pistorius and Yor (2014)). The valuation methods give rise to nonlinear expectation operators that are solutions to nonlinear partial integro differential equations. The underlying mathematical structure of working with the optimal control of such equations in continuous time is under development and not yet available. We seek here instead to develop dynamic conic hedging in discrete time using in addition short maturity options aligned with the time step to analyse the competitive benefits that arise.

The questions addressed include the deviation of the delta or position in the underlying risk for both the hedging of bid and ask values from the derivative of the corresponding bid and ask value functions and an analysis of the factors influencing these departures. For example we observe that in left skewed markets, deltas for strangles rise above the value function derivatives, while they fall if the market is right skewed. Furthermore we evaluate the additional benefits in terms of competitiveness of also allowing for dynamic conic gamma hedging and dynamically hedging the exposure to the skewness. Also reported on are dynamic hedging using short maturity, $5 \%$ out of the money, put and call options.

The concept of dynamic conic delta and gamma hedging introduced here differs fundamentally from risk management approaches aimed at delta and or gamma neutrality. The latter seek to zero out certain derivatives of the current value function. We seek to add positions to future value functions that enhance current market values. In fact the literature has asked and investigated whether the risk management activities of delta and or gamma neutrality enhance firm value (see for example Allayannis and Weston (2001), Jin and Jorion (2006), Adam and Fernando (2006), Bartram, Brown and Conrad (2011)). Our perspective 
on hedging is that it is only to be done with a view to raising the bid price of assets or to lower the ask price of liabilities and hence enhance firm value as defined for example in Madan and Schoutens (2011). Hedging impacts firm value in two price economies by improving the quality of the residual risk held by the firm as reflected in a higher valuation of its assets at bid and a lower valuation of hedged liabilities at the ask.

Under the law of one price and complete markets, on the other hand, we have the irrelevance of hedging and the perfect capital markets of Modigliani and Miller (1958). This irrelevance is a consequence of the linearity of valuation in such economies when they are arbitrage free (Ross (1978)). As a consequence the market value of a portfolio is just the sum of component valuations and there are no benefits to either diversification or hedging in the market value. In classical approaches the benefits of diversification and hedging as expounded for example in Markowitz (1952) or Merton (1971), appear in the personalized utility valuations of investors and not in the market valuations of portfolios. For these benefits to occur in market valuations one needs to work with valuation concepts that permit or reflect advantages to diversification and hedging in order to distinguish between different portfolios. Given that actual corporate positioning in such activities is substantial as noted in Campello, Lin, Ma and Zou (2011), one is led to question the supposed linearity of valuations. Most assets and liabilities of major corporates can hardly be regarded as priced in liquid markets under the law of one price. A two price approach, with its limitations recognized, may then provide a closer and more realistic approximation to market realities.

In the absence of replication based hedging one may evaluate for each product being hedged the quality of hedging instruments. For example we show in hedging a strangle or a capped strangle that dynamic gamma hedging is superior to taking dynamic positions in at the money straddles. Among the hedges reported dynamic hedging with $5 \%$ out of the money put and call options is the most competitive for hedging strangles. The availability of residual risk and the sensitivity of the bid ask spread to this risk helps in selecting and designing hedging strategies. In addition we note as a benchmark case, that if the underlying risk has a Gaussian distribution then dynamic conic gamma hedging can approximate closely the law of one price by essentially reducing the spread to zero. In summary, this paper provides a new hedging methodology, coupled with a quantitative analysis of the impact of hedge designs on firm value leading to educated choices of hedging instruments that have a wide range of applications for financial as well as nonfinancial firms.

The outline of the rest of the paper is as follows. Section 2 sets out the broad design for the study of dynamic conic hedging of claims. Section 3 presents conic hedging in the simpler context of binomial and trinomial trees, where if the number of hedging assets are one or two respectively, residual risks are absent and we are back to complete markets with zero spreads. Section 4 develops the multinomial approximation to the Lévy process employed in a case study illustrating the dynamic conic hedging of simple options. Section 5 provides the details for the construction of the dynamic conic hedge as a discounted nonlinear expectation for such a multinomial evolution. Section 6 presents results on implementing the hedge policies. 
Section 7 presents a comparison of valuations and deltas for the capped strangle and for the sum of its two components of a capped put and a capped call taken separately. Section 8 concludes.

\section{Dynamic Conic Hedging}

Dynamic conic hedging is developed in this section for a discrete time model with a time step of $h$. For ease of discussion and notation illustrative applications model the underlying risk, on which claims are written, to be the one dimensional process for the price of a stock or stock index observed in discrete time with value $S(t)$ at time $t$. The interest rate will be constant at the continuously compounded rate of $r$ percent per annum. The base probability law for the underlying stock price evolution will be a risk neutral Markovian law. Furthermore, all hedging assets in addition to the stock are of the shortest maturity corresponding to the time tenor. The stock and all hedging assets are priced under this risk neutral law with one price for trading in both directions. An extension allowing for transaction costs for the so called liquid assets, permitting directional on the hedging assets could be further developed using the methods of Jouini and Kallal (1995), Cvitanic and Karatzas (1996), Kabanov (1999) and Schachermayer (2004). For the purposes of this paper such developments are left for subsequent research and hedging assets are treated as liquid with no spreads.

Excluding the hedging assets, other financial products or contingent claims are supposed to be traded in a two price economy. Such economies have two value functions for the market value of cash flows that are yet to be realized. These cash flows are the remaining cash flows at the time of valuation. The two values are given by ask and bid value functions $V_{A}(t), V_{B}(t)$ with $V_{A}(t) \geq V_{B}(t)$. This section develops the basic equations for these valuation functions for arbitrage free two price economies. We note however, that under certain assumptions, the two valuation functions can be reduced to just the bid pricing function. It is shown in Madan (2014a) that when the set of traded cash flows are closed under negation then one may infer ask prices as the negative of the bid price for a claim to the negative. Essentially, with $X \geq 0$ for example, buying $-X$ is tantamount to delivering or selling $X$. The resulting negative bid price for $-X$, is then what one must be paid for the sale. Hence the negative of this negative bid is the ask price. Assuming such closure under negation, for the traded assets, one needs to develop the equations for just the bid price.

What, we may ask, are the observable counterparts in actual markets, to the theoretical constructs of bid and ask prices in two price economies. They are meant to be prices for selling to, or buying from market, a variety of state contingent future cash flows. Importantly, the counterparty here for all transactions, is an abstract market that stands ready to trade in both directions at its two prices. The market structure is thereby comparable to that of an Arrow Debreu equilibrium except that now the market price also depends on the trade direction. Consider now a variety of claims like portfolios of options, some structured 
products, or insurance contracts. There may not be an active market delivering observable transaction prices at any regular frequency for such complex claims. Yet one could with some effort obtain quotes at which they could be bought or sold. Furthermore, even for relatively liquid assets like stocks, where there are transaction prices, one could consider these as valuations, and take for a bid price over a discrete interim of time, the lowest transaction price in this interval as a conservative valuation. Similarly the highest transaction price would serve as a conservative estimate for the price at which the asset could have been secured. Such maxima and minima of transaction prices would have the structure of bid and ask prices constructed in two price economies. For example the bid for a portfolio of stocks should be higher than the sum of the bids for the components. Madan (2014b) on employing such conservative valuation procedures, using data on stock prices, reports portfolio premia of around $4 \%$ for the quarterly period. Somewhat coarser observations on conservative valuations may be obtained from the haircuts experienced by equity offered as collateral in repo markets. These can range from 5 to 15 percent depending on the type of equity, as reported by ICMA (2012).

For such two price economies, with their conservative valuations, hedging mechanisms that deliver an increase in bid prices, and or a decrease in ask prices are then to be seen as more competitive, leading to markets with lower spreads. Two price economies employing the same pricing functional on the same product, can lower price spreads by using different and more efficient hedges. The lower spreads imply a more competitive market structure from the perspective of the investing and trading community.

Consider now a general state contingent cash flow with a post hedge claim to $(c(t), t=$ $h, \cdots, N h)$. In a two price economy such claims have two value processes that denoted here by $V_{B}(t)$ and $V_{A}(t)$ for the bid and ask prices for the cash flows to come after time $t$. Madan (2014a) shows that for all two price economies satisfying a no arbitrage condition, the processes valuing future cash flows may be related to the cash flows being valued by a nonlinear valuation operator. Specifically there exists a set $\mathcal{M}$ of positive processes $\theta=$ $(\theta(t), t=1, \cdots, T) \in \mathcal{M}$ such that

$$
\begin{aligned}
V_{B}(t) & =\inf _{\theta \in \mathcal{M}} E_{t}\left[\sum_{u>t} \frac{\theta(u) c(u)}{\theta(t)}\right] \\
V_{A}(t) & =\sup _{\theta \in \mathcal{M}} E_{t}\left[\sum_{u>t} \frac{\theta(u) c(u)}{\theta(t)}\right] .
\end{aligned}
$$

The expectation operator $E_{t}[\cdot]$, here is under a base probability that in the applications below will be taken to be a base risk neutral probability choice with respect to the hedging assets being employed. Under some further conditions related to maintaining dynamic time consistency the valuation operators can be taken to be nonlinear conditional expectation operators described, for example, by Cohen and Elliott (2010) in a discrete time context. 
One may then write

$$
\begin{aligned}
& V_{B}(t)=\inf _{\theta \in \mathcal{M}_{t}} E_{t}\left[\frac{\theta(t+h)\left(c(t+h)+V_{B}(t+h)\right)}{\theta(t)}\right] \\
& V_{A}(t)=\sup _{\theta \in \mathcal{M}_{t}} E_{t}\left[\frac{\theta(t+h)\left(c(t+h)+V_{A}(t+h)\right)}{\theta(t)}\right],
\end{aligned}
$$

where $\mathcal{M}_{t}$ is the restriction of $\mathcal{M}$ with respect to just the choices for $(\theta(t), \theta(t+h))$. As noted already many hedging objectives like variance or semivariance are not time consistent. Dynamically time consistent objectives have the useful property that a hedging plan solved for at an earlier date for a later date is still what one wants to do when we get to the later date. Later in the paper this optimality principle is established, for the particular nonlinear conditional expectation we employ. Note additionally that classical conditional expectation operators are linear and hence ill suited for optimization objectives. In contrast, nonlinear conditional expectation operators are concave for the bid price via the infimum while the ask price is convex via the supremum. The former we seek to maximize and the latter we minimize. Furthermore, they naturally arise by no arbitrage in two price economies and we employ them here as our hedging objectives. Henceforth we synthesize the one step ahead cash flow plus remaining value as

$$
\begin{aligned}
& X_{B}(t+h)=c(t+h)+V_{B}(t+h), \\
& X_{A}(t+h)=c(t+h)+V_{A}(t+h) .
\end{aligned}
$$

For a sharper understanding of the pricing rules, characterizing all arbitrage free two price economies, one has to inquire into the nature of the positive processes $\theta$ that defining the two valuations. Madan (2014a) decomposes the positive processes $\theta$, arising from no arbitrage into the product of a discount function and a measure change martingale. They are then identical to what are popularly called pricing kernels (Cochrane (2001)). The infimum over such processes $\theta$, then allows for interactions between the local discount rate and the severity of the measure change. The result then incorporates multicurve discounting that is now also a standard in the industry. However, for simplicity, here we assume that loans are priced in a liquid market under the law of one price with the continuously compounded interest rate of $r$. One then observes that when $X_{B}(t+h)$ is identically unity, the bid and ask price at time $t$ for the pure discount bond of maturity $t+h$ is $e^{-r h}$ and furthermore by equality of bid and ask, it is the case that for all $\theta \in \mathcal{M}$

$$
e^{-r h}=E_{t}\left[\frac{\theta(t+h)}{\theta(t)}\right] .
$$


One may then write

$$
V_{B}(t)=e^{-r h} \inf _{\theta \in \mathcal{M}_{t}} E_{t}\left[\frac{\theta(t+h)}{E_{t}[\theta(t+h)]} X_{B}(t+h)\right] .
$$

Equation (1) expresses the bid price as a discounted infimum of expectations under a collection of one step ahead measure changes with the measure change given by

$$
M_{\theta}(t+h)=\frac{\theta(t+h)}{E_{t}[\theta(t+h)]} \text { for } \theta \in \mathcal{M}_{t} .
$$

We may associate with each one step ahead change of measure density $M_{\theta}(t+h)$ a change of probability $Q^{\theta}$ and then write

$$
V_{B}(t)=e^{-r h} \inf _{\theta \in \mathcal{M}_{t}} E_{t}^{Q^{\theta}}\left[X_{B}(t+h)\right]
$$

The set of measures $Q^{\theta}$ associated to processes $\theta \in \mathcal{M}_{t}$ allow for variations in the market prices of systematic risk along with variations in the possible set of systematic factors that may relevant at any time, in defining a conservative market value. This aspect is commented on further in Section 2.2 below.

We now introduce into this context a finite set of hedging assets. Consider the availability of a set of $m$ financed zero cost hedging instruments that mature at the next time step with the payoff $H(t+h)=\left(H_{1}(t+h), \cdots, H_{m}(t+h)\right)$. Under the zero cost assumption we have that

$$
E_{t}[H(t+h)]=0 \text {. }
$$

By assumption (3) the base measure is a risk neutral choice. Also the hedging assets, when they include options, have as the only relevant prices in defining the cash flows to these assets, their current market prices. Zero cost is then organized by borrowing the price and paying it back with interest at the maturity which is the next time step. At this next time step the value is known by the payoff. With some additional complexity in the modeling one may allow for bid and ask prices for these short maturity options employed in hedging longer dated claims. We abstract from these considerations for simplicity of exposition.

Dynamic conic hedging is concerned with taking positions in the hedging assets with a view to locally, maximizing the bid price or minimizing the ask price, of the hedged position. Though the objective function for this exercise is concave for the bid and convex for the ask it does scale with the cash flows involved and hence the objective could potentially be unbounded with no interior solution to the hedging problem. However, under fairly general conditions on the structure of the problem we are assured of an interior solution. Recall that acceptability of a cash flow requires a nonnegative bid price for the cash flow. 
Definition 1 The set of acceptable risks is said to be conservative if the cone of acceptable risks is strictly contained in the half space associated with a classical risk neutral measure.

We suppose that $\theta^{0}(t)=\exp (-r t) \in \mathcal{M}$ and hence the base measure is $Q^{\theta^{0}}$ and hence the cone of acceptability is conservative.

Definition 2 The set of hedging assets is said to be admissible for a cone of acceptability defined by the measures associated with the processes $\theta \in \mathcal{M}$ if

$$
\zeta=\sup _{\eta,\|\eta\|=1} \inf _{\theta \in \mathcal{M}} E_{t}^{Q^{\theta}}\left[\eta^{T} H(t+h)\right]<0
$$

The hedging assets have a zero risk neutral expectation and so all portfolios. The infimum over measures in $\mathcal{M}$ should then be negative or at least nonpositive. The admissibility condition essentially states one cannot form a portfolio of the hedging assets that becomes close to acceptable on its own. Acceptability is the generalization of arbitrage and one should not be able to get close to such at zero cost. The acceptable strategies are restricted to hedge positions $\eta$ being adapted to information available at time $t$. They are selffinancing as the investments here are in zero cost cash flows.

Definition 3 The set of one step ahead target cash flows $X_{B}(t+h)$ are said to be hedgeable if

$$
\sup _{\theta \in \mathcal{M}} E_{t}^{Q^{\theta}}\left[\left|X_{B}(t+h)\right|\right] \leq A<\infty
$$

Certainly bounded one step ahead cash flows are hedgable. More generally allowing for unbounded cash flows we ask for a uniform bound for all the $L_{1}$ norms associated with the test measures $\mathcal{M}$.

Proposition 4 Assume we have a conservative set of acceptable risks, an admissible set of hedging assets for this cone and a hedgeable target cash flow then the dynamic conic hedging bid problem given by

$$
\widetilde{V}_{B}(t)=\sup _{a} e^{-r h} \inf _{\theta \in \mathcal{M}} E_{t}^{Q^{\theta}}\left[X_{B}(t+h)+a^{T} H(t+h)\right]
$$

has a solution given by

$$
a^{*}=\arg \sup _{a} e^{-r h} \inf _{\theta \in \mathcal{M}} E_{t}^{Q^{\theta}}\left[X_{B}(t+h)+a^{T} H(t+h)\right] .
$$


Proof. Let $\lambda=\|a\|$ and write

$$
\widetilde{V}_{B}^{\lambda}(t)=\lambda e^{-r h} \sup _{\eta,\|\eta\|=1} \inf _{\theta \in \mathcal{M}} E_{t}^{Q^{\theta}}\left[\frac{1}{\lambda} X_{B}(t+h)+\eta^{T} H(t+h)\right]
$$

For $\varepsilon>0$ there exists $\eta$ such that

$$
\lambda e^{-r h} \inf _{Q^{\theta}} E_{t}^{Q^{\theta}}\left[\frac{1}{\lambda} X_{B}(t+h)+\eta^{T} H(t+h)\right]>\widetilde{V}_{B}^{\lambda}(t)-\varepsilon \lambda e^{-r h}
$$

Now

$$
\begin{aligned}
E_{t}^{Q^{\theta}}\left[\frac{1}{\lambda} X_{B}(t+h)+\eta^{T} H(t+h)\right] & =\frac{1}{\lambda} E_{t}^{Q^{\theta}}\left[X_{B}(t+h)\right]+E_{t}^{Q^{\theta}}\left[\eta^{T} H(t+h)\right] \\
& \leq \frac{A}{\lambda}+E_{t}^{Q^{\theta}}\left[\eta^{T} H(t+h)\right]
\end{aligned}
$$

So

$$
\begin{aligned}
\inf _{Q^{\theta}} E_{t}^{Q^{\theta}}\left[\frac{1}{\lambda} X_{B}(t+h)+\eta^{T} H(t+h)\right] & \leq \frac{A}{\lambda}+\inf _{Q^{\theta}} E_{t}^{Q^{\theta}}\left[\eta^{T} H(t+h)\right] \\
& \leq \frac{A}{\lambda}+\sup _{\eta,\|\eta\|=1} \inf _{Q^{\theta} \in \mathcal{M}} E_{t}^{Q^{\theta}}\left[\eta^{T} H(t+h)\right] \\
& =\frac{A}{\lambda}+\zeta
\end{aligned}
$$

It follows that

$$
\lambda e^{-r h} \inf _{Q^{\theta}} E_{t}^{Q^{\theta}}\left[\frac{1}{\lambda} X_{B}(t+h)+\eta^{T} H(t+h)\right] \leq A e^{-r h}+e^{-r h} \lambda \zeta
$$

Hence

$$
\widetilde{V}_{B}^{\lambda}(t)<A e^{-r h}+e^{-r h} \lambda(\zeta+\varepsilon)
$$

For $\varepsilon<|\zeta|$ we have that $\widetilde{V}_{B}^{\lambda}(t) \rightarrow-\infty$ as $\lambda \rightarrow \infty$. Therefore the supremum over $a$ will occur in the interior of a compact set and $a^{*}$ is well defined.

Corollary 5 The dynamic conic hedging ask problem defined by

$$
\widetilde{V}_{A}(t)=\inf _{a} e^{-r h} \sup _{\theta \in \mathcal{M}} E_{t}^{Q^{\theta}}\left[X_{A}(t+h)+a^{T} H(t+h)\right]
$$


also has a solution defined by

$$
a^{*}=\arg \inf _{a} e^{-r h} \sup _{\theta \in \mathcal{M}} E_{t}^{Q^{\theta}}\left[X_{A}(t+h)+a^{T} H(t+h)\right] .
$$

The ask problem is solved on solving the bid problem for $-X_{A}$.

Proposition 6 If the target cash flow can be perfectly hedged by replication then the conic hedge is this replication hedge.

Proof. Suppose for the target cash flow $X$ one may position in the hedging assets to attain

$$
\alpha-X
$$

It follows that

$$
E_{t}[X]=\alpha
$$

furthermore for any attainable hedge cash flow $Y$ we have that

$$
\inf _{\theta \in \mathcal{M}} E_{t}^{Q^{\theta}}(X+Y) \leq E_{t}[X+Y]=\alpha
$$

The presentation and discussion so far has been at the level of adaptive processes with values and associated hedges being dependent in principle on the full time paths of observable variables up to and including time $t$. This is fine from a general theoretical perspective but for explicit computations one needs to work in a manageable Markovian dimension. Our illustrations and computations will be conducted in the context of a one dimensional Markovian model for the stock price with hedge positions that will be just functions of the stock price and time.

For an arbitrary Markovian risk neutral evolution and cash flows defined by a cash flow stream $c(t)=g(S(t), t)$ over discrete time at the time step $h$ for $t=k h$ for $k=1, \cdots, N$ and $T=N h$ one may define the bid and ask values for the remaining uncertainty recursively by

$$
\widetilde{V}_{B}(S(T), T)=\widetilde{V}_{A}(S(T), T)=g(S(T), T) .
$$

We then work backwards across the time grid and over the space variate to build the dynamically conically hedged bid and ask price processes $\widetilde{V}_{B}(S(t), t)$ and $\widetilde{V}_{A}(S(t), t)$. This is done sequentially, by solving the one step ahead dynamic conic hedging bid and ask problems. In the case reported on later in the paper we consider a variety of hedging assets, that include the stock, the one step ahead squared return on the stock or the realized forward gamma, the one step ahead realized skewness or the realized forward skew and the use of one step ahead out of the money put and call options. 


\subsection{Comonotone Additivity and Law Invariance}

With a view towards the development of a specific valuation functional we introduce two additional hypotheses on these functionals. They are termed comonotone additivity and law invariance.

\subsubsection{Comonotone Additivity}

The bid pricing functional, in a two price economy, for a one step ahead risk exposure $X_{B}(t+h)$, that may include a hedge position is defined by

$$
b\left(X_{B}(t+h)\right)=\inf _{\theta \in \mathcal{M}_{t}} E_{t}^{Q^{\theta}}\left[X_{B}(t+h)\right] .
$$

An important property of the bid pricing functional is that of additivity for comonotone risks.

Definition 7 Two random variables are said to be comonotone if they have no negative comovements or almost surely for the product measure on $\left(\omega_{1}, \omega_{2}\right)$ we have

$$
\left(X\left(\omega_{1}\right)-X\left(\omega_{2}\right)\right)\left(Y\left(\omega_{1}\right)-Y\left(\omega_{2}\right)\right) \geq 0 .
$$

Definition 8 The bid price functional is comonotone additive if for $X, Y$ comonotone we have that

$$
b(X+Y)=b(X)+b(Y)
$$

Under comonotone additivity there are no diversification benefits for comonotone risks and we employ comonotone additive bid and hence ask price functionals. Schmeidler (1986) showed that such bid price functionals are comonotone additive if and only if the set of measures associated with the defining set of processes $\theta \in \mathcal{M}_{t}$ is the core of a convex game and we refer to Schmeidler(1986) and Delbaen (2000) for further details on these matters. Essentially the bid price may be seen as an integral with respect to a non-additive measure or probability. When this underlying non-additive probability is convex, i.e. satisfies the condition that the measure of the union of two sets plus the measure of the intersection dominates the sum of the measures then we have comonotone additivity. The convexity of the probability is related to the presence of risk aversion (Chateauneuf (1991, Yaari (1987)). Of course the risk aversion being modeled here is that of the market as embedded in its convex set of acceptable risks.

We note that if the bid price is comonotone additive and there is a portfolio $a^{T} H$ that is comonotone with the target $X$ then adding a positive multiple $\lambda$ of this portfolio will lower the bid price as

$$
b\left(X+\lambda a^{T} H\right)=b(X)+\lambda b\left(a^{T} H\right) \leq b(X)+\lambda|a| \zeta<b(X),
$$


by definition 2 .

The property of comonotone additivity is also useful in dealing with swap contracts or contracts with signed cash flows that may move from the asset to the liability side of the balance sheet depending on circumstances. Under the law of one price suppose the remaining uncertainty provides access to a random and signed cash flow $X$. We may then write

$$
X=X^{+}-X^{-}
$$

and report positive value of $X^{+}$as an asset on the asset side of the balance sheet while we report the positive value of $X^{-}$on the liability side as a liability. Under comonotone additivity we have that $X^{+}$and $\left(-X^{-}\right)$are comonotone and hence if we report as an asset we have

$$
\begin{aligned}
b(X) & =b\left(X^{+}\right)+b\left(-X^{-}\right) \\
& =b\left(X^{+}\right)-a\left(X^{-}\right)
\end{aligned}
$$

Equivalently when reporting as a liability we have

$$
\begin{aligned}
a(X) & =a\left(X^{+}\right)+a\left(-X^{-}\right) \\
& =a\left(X^{+}\right)-b\left(X^{-}\right)
\end{aligned}
$$

so either way we may report the positive part as an asset at bid and the negative part as a liability.

However when it comes to taking hedge positions we cannot hedge $X^{+}$and $X^{-}$separately with two different hedge positions for they will have to be put together and the aggregate position will not be optimal for $X$. So the hedge must be worked out for $X$ as $a^{T} H$ and then one may report $\left(X-a^{T} H\right)^{+}$as an asset while $\left(X-a^{T} H\right)^{-}$is reported as a liability. When working backwards recursively with an intermediate cash flow $c$ coupled with the value of the remaining uncertainty say $b$ one defines $X=c+b$ and then one may split the post hedge reporting onto the two sides for comonotone additive pricing functionals.

\subsubsection{Law invariance}

A second important property of the bid price functional is that of law invariance.

Definition 9 The bid price functional is said to be law invariant if the bid price is just a function of the probability law or if for two random variables $X, Y$ we have that for all a

$$
\operatorname{Pr}(X \leq a)=\operatorname{Pr}(Y \leq a)
$$

then $b(X)=b(Y)$. 
Kusuoka (2001) showed that for law invariant and comonotone additive bid price functionals there exists a concave distribution function $\Psi(u), 0 \leq u \leq 1$ defined on the unity interval such that with $F_{X}(x)$ the distribution function of $X$ we have

$$
b(X)=\int_{-\infty}^{\infty} x d \Psi\left(F_{X}(x)\right)
$$

The case studies we report on employ such law invariant and comonotone additive bid and ask price functionals. The pricing functional (4) has connections with utility theory and it is shown in Madan (2014b) that this functional is precisely that obtained from the dual theory of choice of Yaari (1987). The set of supporting measures is also compared to the good deal bounds of Cochrane and Saa-Requejo (2000), by Madan (2014b) where it is shown that good deals are acceptable at an appropriately constructed level.

We note in this regard that in economies in general the resulting two price equilibria may involve an evaluation of multidimensional probability laws of a risk and its covariation with other systematic risk components. Such a generalization will involve the use of multivariate risk measures as studied for example in Yaari (1986) and Galichon and Henry (2012). Leaving these developments for future research, we focus attention here on the simpler case of a one dimensional probability law. A market populated with a diverse set of new entrants with varying or even no prior exposures may well employ just a one dimensional probability law. On the other hand, the information requirements of multidimensional laws may be too demanding, thereby forcing the economy to use just the marginal law.

An alternative interpretation of the objective function proposed here is the maximization of a risk adjusted value using a nonlinear expectation as a dynamic risk measure. From such a perspective the parameters of the objective may be calibrated to the risk attitudes of the investor or entity for whom the hedge is being designed. The valuation operators under such an interpretation may be further generalized in the direction of incorporating ambiguity aversion at a personal level as studied in Gilboa and Schmeidler (1989) or Klibanoff, Marrinacci and Mukerji (2005). They could even be altered further to incorporate the mathematical representation of risk preferences as taken up in behaviorial finance and studied in Jin, Xu and Zhou (2008) for example. However, these are directions of research refining the formulation of personal risk attitudes while our purpose here is to present an arbitrage free market valuation perspective, instead that places different restrictions on the set of possibilities that may be entertained. For example, in behavioral finance one may consider $\mathrm{S}$ shaped distortions while no arbitrage in two price economies forces the distortions to be concave.

Hedging exercises reported later in the paper develop multinomial approximations for exponential Lévy risk neutral stock price processes, working specifically here with the variance gamma process. For any finite state evolution the integral (4) is a finite sum given in terms of the ordered sequences $V_{B}(t+h)_{(k)}$ and the associated ordered sequence of probabilities 
$\pi_{(k)}$.

$$
V_{B}(S(t), t)=\sum_{k} V_{B}(t+h)_{(k)}\left(\Psi\left(\sum_{l \leq k} \pi_{(k)}\right)-\Psi\left(\sum_{l<k} \pi_{(k)}\right)\right) .
$$

For each time step on a space time grid and for each value on the space grid one may implement the recursion (5) to evaluate the unhedged bid price across the entire space time grid after choosing the distortion $\Psi$. For the ask price we merely evaluate the negative of the bid price for the negative. The values at maturity are given by the payout in both cases. The recursion (5) is for the unhedged claims.

\subsection{The Optimality Principle for Nonlinear Expectations based on distortions}

For a finite horizon discrete time optimization let the control variables be denoted $\left\{y_{t}\right\}$ with state variables $\left\{x_{t}\right\}$ and let the transition functions be given by

$$
x_{t+1}=h_{t}\left(x_{t}, y_{t}, u_{t+1}\right) \text {. }
$$

The variables $u_{t+1}$ are the random components. For a feasible policy we may specify a constraint set as

$$
y_{t} \in \mathcal{A}\left(x_{t}\right)
$$

We wish to consider a value function given as the nonlinear expectation using distortions, of a function of the final state. We define the value at maturity as

$$
W^{(y)}\left(x_{T}, T\right)=G\left(x_{T}\right)
$$

For all other $t$, given a feasible policy $y$ suppose we have defined $W^{(y)}\left(x_{t+1}, t+1\right)$ we then define

$$
W^{(y)}\left(x_{t}, t\right)=\int_{-\infty}^{\infty} v d \Psi\left(\operatorname{Pr}\left(W^{(y)}\left(h_{t}\left(x_{t}, y_{t}, u_{t+1}\right), t+1\right) \leq v\right)\right) .
$$

We wish to find a policy $y$ that maximizes this value function at time 0 . Abstracting from technical considerations suppose that states, actions and random elements are finite.

For the optimality principle define the backward recursion solution whereby

$$
\begin{aligned}
V(x, t) & =\max _{y \in \mathcal{A}(x)} \int_{-\infty}^{\infty} v d \Psi(\operatorname{Pr}(V(h(x, y, u), t+1) \leq v)) \\
V(x, T) & =G(x) .
\end{aligned}
$$


We also define $\kappa_{t}(x)$ by

$$
V(x, t)=\int_{-\infty}^{\infty} v d \Psi\left(\operatorname{Pr}\left(V\left(h\left(x, \kappa_{t}(x), u\right), t+1\right) \leq v\right)\right)
$$

Consider the global problem of finding functions $\mu_{t}(x), t=0, \cdots, T-1$ to maximize

$$
W(x, 0)
$$

defined by the recursion

$$
W(x, T)=G(x)
$$

and

$$
\begin{aligned}
W(x, t) & =\int_{-\infty}^{\infty} v d \Psi\left(\operatorname{Pr}\left(W\left(h\left(x, \mu_{t}(x), u\right), t+1\right) \leq v\right)\right) \\
& \geq \int_{-\infty}^{\infty} v d \Psi\left(\operatorname{Pr}\left(W\left(h\left(x, \kappa_{t}(x), u\right), t+1\right) \leq v\right)\right) .
\end{aligned}
$$

Suppose inductively that $W(x, t+1)=V(x, t+1)$. It follows that

$$
\begin{aligned}
W(x, t) & =\int_{-\infty}^{\infty} v d \Psi\left(\operatorname{Pr}\left(V\left(h\left(x, \mu_{t}(x), u\right), t+1\right) \leq v\right)\right) \\
& \leq V(x, t)
\end{aligned}
$$

Also we have

$$
\begin{aligned}
V(x, t) & =\int_{-\infty}^{\infty} v d \Psi\left(\operatorname{Pr}\left(W\left(h\left(x, \kappa_{t}(x), u\right), t+1\right) \leq v\right)\right) \\
& \leq W(x, t)
\end{aligned}
$$

It follows that $W(x, t)=V(x, t)$ and the backward recursion provides the global solution.

\subsection{Conservative Market Values, Systematic Risks and Risk Pre- mia}

We report in this section how distorted risk neutral expectations accommodate systematic risks and their risk premia. Suppose for simplicity we have a systematic factor $S$ and an unpriced and independent residual $U$ making up the risk $X$ in the from

$$
X=S+U
$$


Let the density, under the physical or real world measure of $S, U$ be

$$
p(S) g(U)
$$

Now the real world probability distribution function of $X$ may be computed as

$$
P(X<a)=\int_{-\infty}^{\infty} g(u) d u \int_{-\infty}^{a-u} p(s) d s .
$$

The risk neutral probability however is

$$
\begin{aligned}
Q(X<a) & =\int_{-\infty}^{\infty} g(u) d u \int_{-\infty}^{a-u} q(s) d s \\
& =\int_{-\infty}^{\infty} g(u) d u \int_{-\infty}^{a-u} \lambda(s) p(s) d s
\end{aligned}
$$

where $\lambda(s)$ is the market of risk function.

We may write the difference as

$$
Q(X<a)-P(X<a)=\int_{-\infty}^{\infty} g(u) d u \int_{-\infty}^{a-u}(\lambda(s)-1) p(s) d s
$$

and with $\lambda$ typically decreasing in the systematic factor we see how market prices of risk lift the lower tail of the risk neutral distribution function.

Consider now a distorted expectation of $X$ that has the form

$$
\int_{-\infty}^{\infty} a d \Psi(Q(X<a))=\int_{-\infty}^{\infty} g(u) d u \int_{-\infty}^{\infty} a \Psi^{\prime}\left(\int_{-\infty}^{\infty} g(u) d u \int_{-\infty}^{a-u} \lambda(s) p(s) d s\right) \lambda(a-u) p(a-u) d a
$$

One now observes the distortion as incorporating a change in the market price of risk by the factor

$$
\Psi^{\prime}\left(\int_{-\infty}^{\infty} g(u) d u \int_{-\infty}^{a-u} \lambda(s) p(s) d s\right)
$$

The pricing being undertaken then is a form of conservatism with respect to the market prices of risk. Systematic factors and market prices of risk are incorporated into the distorted risk neutral expectation.

\subsection{Defining Dynamic Acceptability of Risk Exposures}

Risk acceptability was defined locally in Section 2 by a positive distorted expectation. It remains to select the concave distortion and we follow Cherny and Madan (2009) and work 
with the distortion termed minmaxvar by Cherny and Madan (2009) at some stress level $\gamma>0$. Such a distortion reweights large losses upwards to infinity while simultaneously discounting large gains down towards zero. Taken together these features embed both a presence of loss aversion and an absence of gain enticement in the valuation conservatism being modeled into market behaviour. The specific minmaxvar distortion is given by

$$
\Psi^{\gamma}(u)=1-\left(1-u^{\frac{1}{1+\gamma}}\right)^{1+\gamma}
$$

We then have to choose the value of $\gamma$ to be employed at each time step.

For guidance on this choice consider a Gaussian random variable $G$ over the unit time of a year with mean $\mu$ and standard deviation $\sigma$. We may then write

$$
G=\mu+\sigma Z
$$

for $Z$ a standard normal variate. The distorted expectation of $G$, say $\zeta_{G}$ is the given by

$$
\zeta_{G}=\mu-\sigma \zeta_{Z}
$$

where $\zeta_{Z}$ is the negative of the distorted expectation of $Z$. The random variable $G$ is then acceptable provided

$$
\frac{\mu}{\sigma} \geq \zeta_{Z}
$$

For minmaxvar at stress level .25 one may compute $\zeta_{Z}=0.4165$, which makes a Gaussian risk with a volatility of $15 \%$ acceptable with a mean return of $6.25 \%$. These are reasonable values for the return and volatility of equity markets in the long run and so we could take $\gamma=.25$ for an annualized risk horizon. For a time horizon of $h$ we scale by $h$ and add a small constant level of distortion. We shall present results in a dynamic setting for the choice

$$
\gamma=.01+.25 h
$$

\subsubsection{Further remarks on market calibrations of the distortion stress parameter.}

Madan (2014b) estimates the stress level using cross sectional discount factors and add-ons embedded in conservative valuations taken to be the minimal and maximal transaction price for stocks observed over a quarter. The stress level relates the cross sectional quarterly return to the discount and add-on. The implied stress level vary between .2 and .8 with the exception of the crisis when they rose to 1.8. We note additionally that Eberlein and Madan (2009) estimated the highest level of the distortion parameter attained by 527 hedge funds, and the highest percentile reported a value for $\gamma$ of 0.7887 , with a median value 0.2495 and an interquartile range of 0.1673 to 0.3529 . These parameters were also estimated by Cherny and Madan (2010) on market bid and ask prices of options on the $S \& P 500$ index, attaining 
a highest value for $\gamma$ of 0.1774 at the peak of the financial crisis in September 2008 .

In a study on the effects of stress levels on the leverage being provided to an economy measured by the ratio of the scale of operations to the capital being required, Carr, Madan and Vicente-Alvarez (2011) observe that $\gamma$ levels above 0.4405 remove all leverage for Gaussian risks. Eberlein and Madan (2012) show in a Gaussian model for assets with firm value reflecting the benefits of limited liability that a $\gamma$ level of 0.22 equates the sensitivity to volatility of capital required to the sensitivity of firm value to the same. There are then a variety of ways to market calibrate from theoretical and empirical approaches an appropriate stress level.

\section{The Case of Binomial and Trinomial Trees}

For an initial exposition of conic hedging we present results in the simpler context of binomial and trinomial trees. Of course we recognize up front that once delta hedging is introduced in the context of binomial trees one has complete markets, with spreads going to zero and bid and ask prices equalling the base risk neutral price. The same conclusion will hold for trinomial trees in the context of say delta and gamma hedging.

\subsection{Conic Pricing for Binomial Trees}

Assume the very simple setting of a one-step binomial tree. The stock price is assumed to be currently at $S_{0}$. We assume an interest rate $r$ and for the simplicitity of the exposition we assume no dividends. The one-step binomial tree model allows the stock price after a time-step of $h>0$ to move to only two possible values, namely $u S_{0}$ and $d S_{0}$, respresenting respectively the up-state and down-state.

In order to exclude arbitrage, we assume $0<d<\exp (r h)<u$. The factors $u$ and $d$ are often chosen in terms of a given (yearly) volatility estimate $\sigma$. A common choice is the so-called Cox, Ross and Rubinstein (1979) setting where

$$
u=\exp (\sigma \sqrt{h}) \text { and } d=\exp (-\sigma \sqrt{h}) .
$$

Consider now a derivative paying out $f_{u} \geq 0$ in the up-state and $f_{d} \geq 0$ in the downstate as in Figure 1.

The current price $f$ of this derivative can be obtained in the risk-neutral setting as the discounted expected payoff under the risk-neutral measure:

$$
f=\exp (-r h)\left(p f_{u}+(1-p) f_{d}\right)
$$



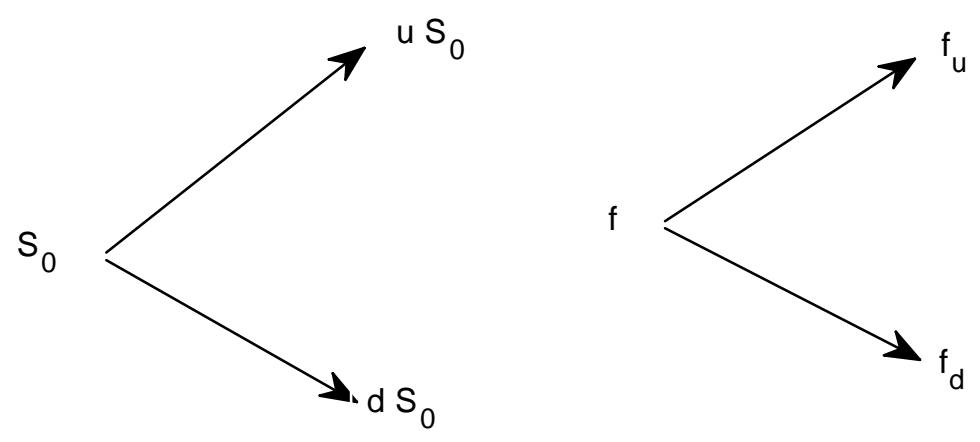

Figure 1: Binomial Tree

where the risk-neutral probability to move to the up-state is given by

$$
p=\frac{\exp (r h)-d}{u-d}
$$

Note that due to our no-arbitrage conditions, this is a proper probability: $0<p<1$.

In a conic setting, we now price using distorted expectations. Assume that $f_{d}<f_{u}$ and recall that risk-neutrally $f_{d}$ is attained with probability $1-p$ and $f_{u}$ with probability $p$. The cdf of the payout is hence represented in Figure 2. It has a jump of size $1-p$ at the point $f_{d}$ and then jumps to the unity level at the point $f_{u}$ with a jump of size $p$. The distorted cdf is also given in the same Figure 2. Hence now under the distortion $f_{d}$ is attained with probability $\Psi^{\gamma}(1-p)$ and $f_{u}$ with probability $1-\Psi^{\gamma}(1-p)$.

We therefore have that

$$
\operatorname{bid}=\exp (-r h)\left(\left(1-\Psi^{\gamma}(1-p)\right) f_{u}+\Psi^{\gamma}(1-p) f_{d}\right),
$$

The ask price is given as the negative of discounted distorted expectation of the negative cash-flow. Since, now $-f_{u}<-f_{d}$ the corresponding cdf is given by a function showing a jump of size $p$ in $-f_{u}$ and a jump of size $1-p$ to unity in $-f_{d}$, as shown in Figure 3 . The distorted cdf has a jump of size $\Psi^{\gamma}(p)$ in $-f_{u}$ and of size $1-\Psi^{\gamma}(p)$ in $-f_{d}$. The ask price is hence given by

$$
\text { ask }=\exp (-r h)\left(\Psi^{\gamma}(p) f_{u}+\left(1-\Psi^{\gamma}(p)\right) f_{d}\right) .
$$

Assume now the other situation where $f_{d}>f_{u}$. Then the risk-neutral cdf first has a jump of size $p$ in the point $f_{u}$ and then jumps to the unity level at the point $f_{d}$ with a jump of size $1-p$. Under distortion $f_{u}$ is attained with probability $\Psi^{\gamma}(p)$ and $f_{d}$ with probability $1-\Psi^{\gamma}(p)$. This is graphed in Figure 4 with values corresponding to the setting for the ATM 


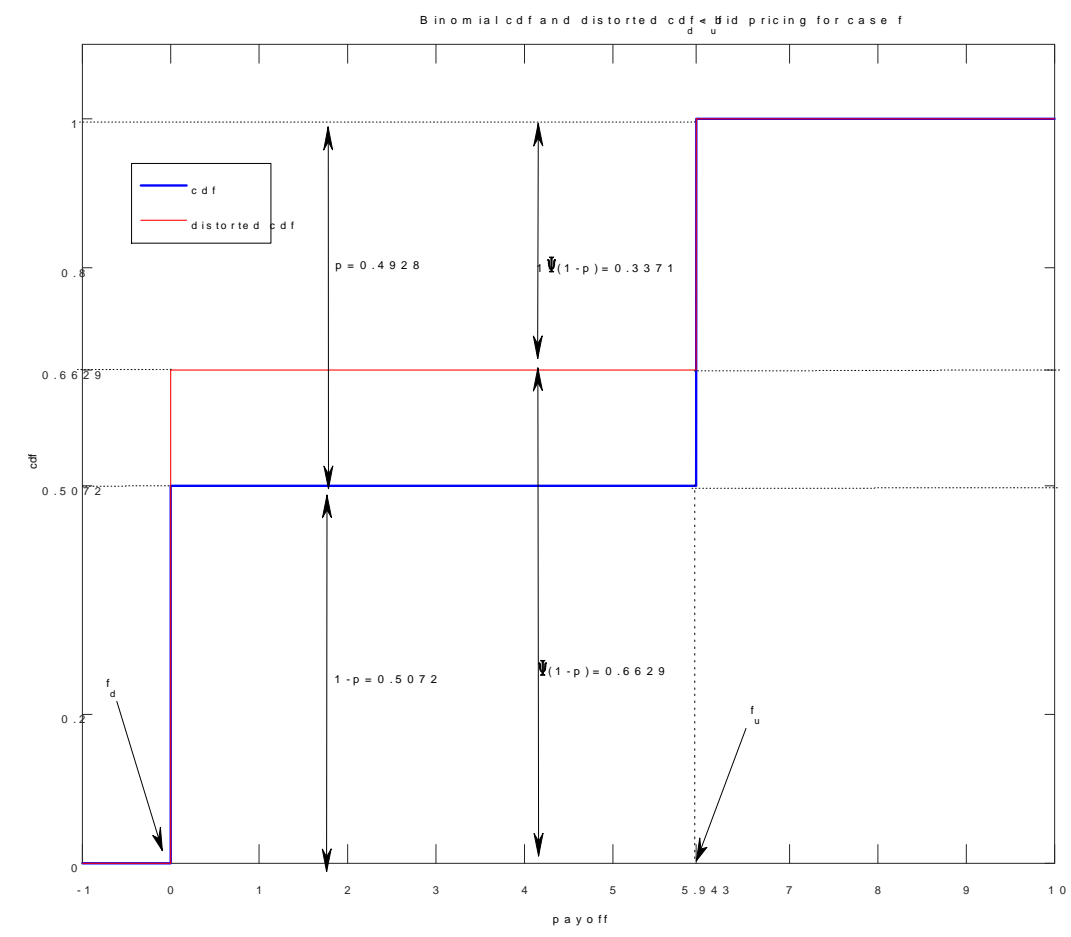

Figure 2: Binomial distribution function and the distorted distribution function. 


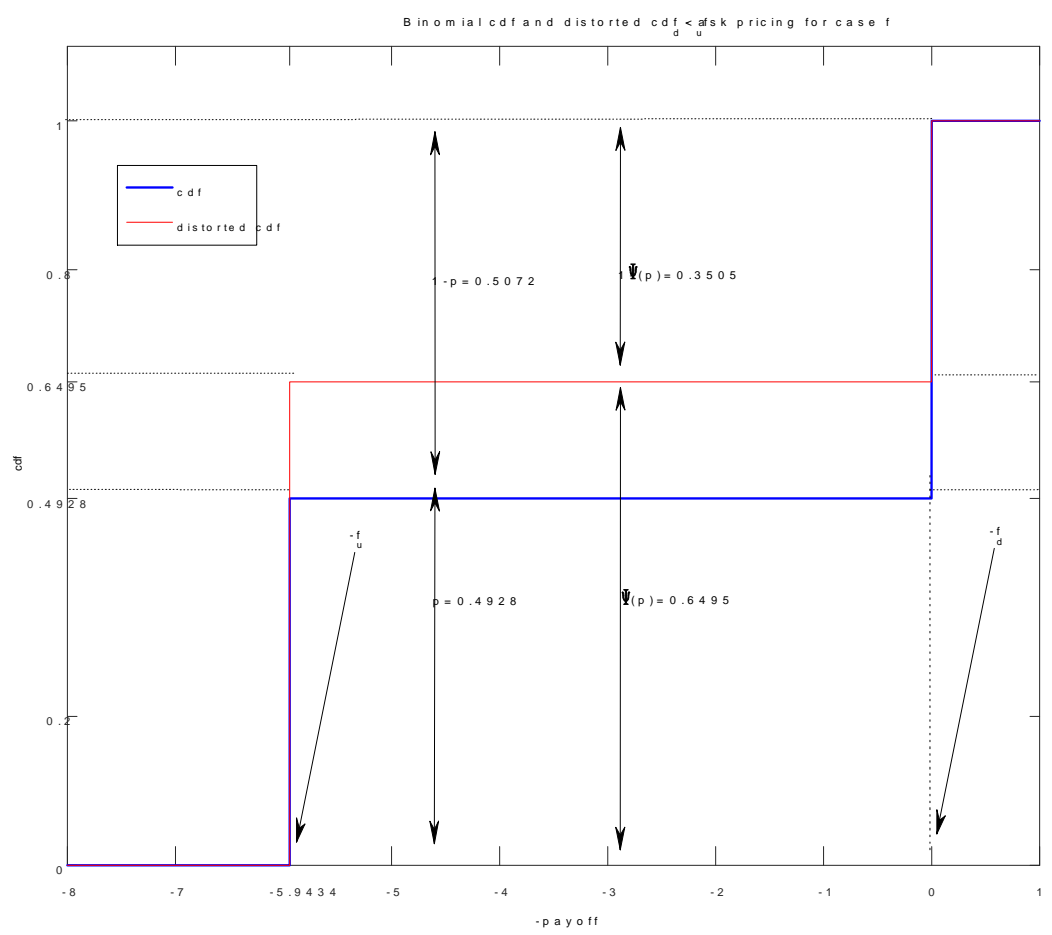

Figure 3: Binomial distribution functions and distortion for ask price. 


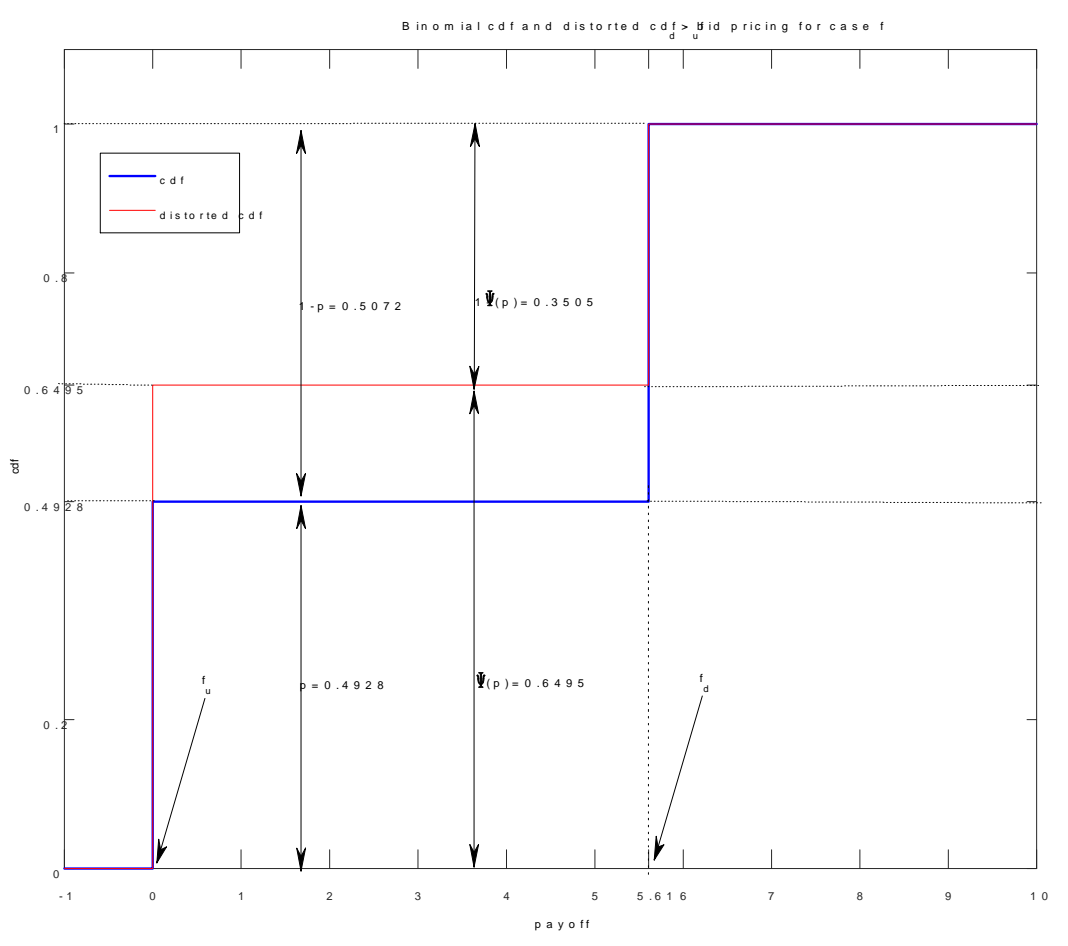

Figure 4: Binomial distribution function with distortion for the reversed case.

put of Example 10). We therefore have that

$$
\operatorname{bid}=\exp (-r h)\left(\Psi^{\gamma}(p) f_{u}+\left(1-\Psi^{\gamma}(p)\right) f_{d}\right),
$$

To determine the ask price, we note that now $-f_{d}<-f_{u}$ and the corresponding riskneutral cdf is given by a function showing a jump of size $1-p$ in $-f_{d}$ and a jump of size $p$ to unity in $f_{u}$, as shown in Figure 5. The distorted cdf has a jump of size $\Psi^{\gamma}(1-p)$ in $-f_{d}$ and of size $1-\Psi^{\gamma}(1-p)$ in $-f_{u}$ as is shown in the same Figure 5 . The ask price is hence given by

$$
\text { ask }=\exp (-r h)\left(\left(1-\Psi^{\gamma}(1-p)\right) f_{u}+\Psi^{\gamma}(1-p) f_{d}\right) .
$$

We summarize the above in the following Table 1, where we provide the distorted probability to reach the up state. The distorted probability to reach the down state is then just one minus this probability.

Example 10 Assume a (yearly) volatility of $20 \%$ and a risk-free interest rate of $1 \%$. We will price a one-month ATM call option. With $\sigma=0.20$ and $h=1 / 12$, we have $u=\exp (\sigma \sqrt{h})=$ 1.059434 and $d=\exp (-\sigma \sqrt{h})=0.943900$. If $S_{0}=100$, we have that $f_{u}=5.9434$ and 


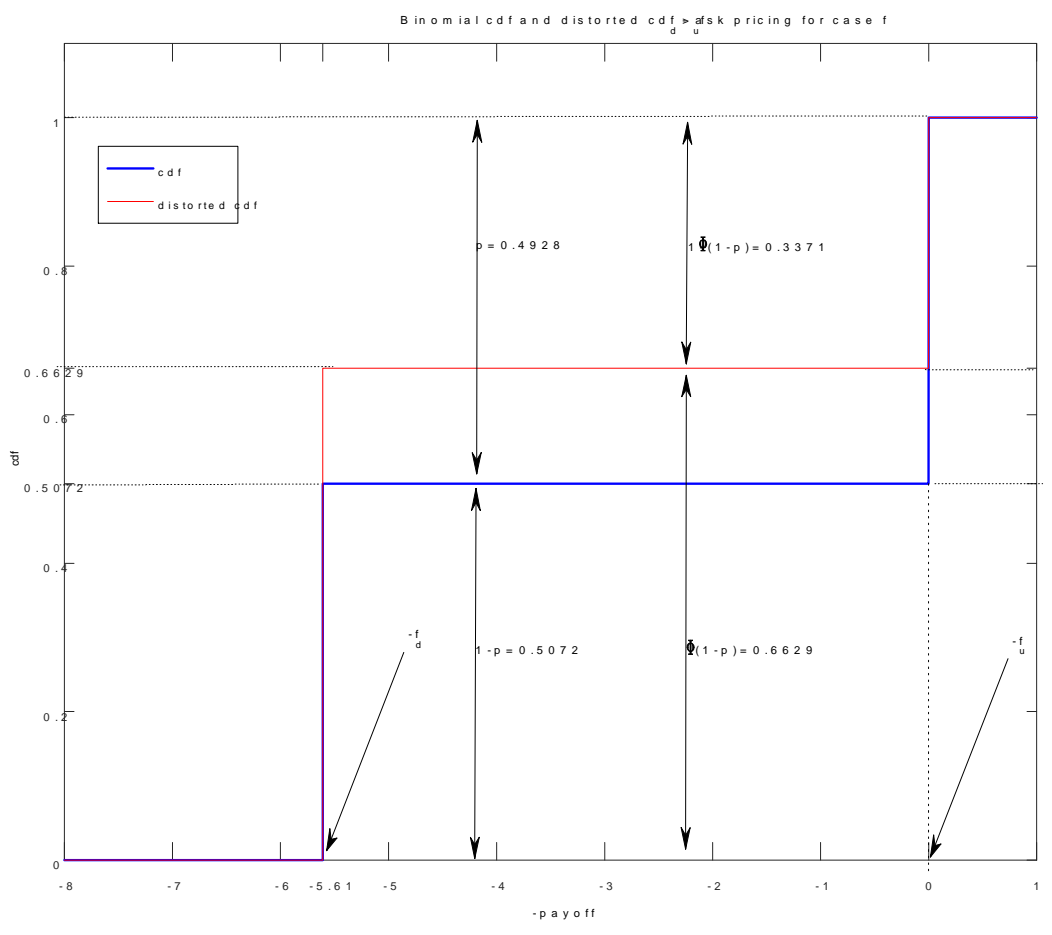

Figure 5: Binomial distribution function and distortion for the reverse case.

\begin{tabular}{c|c|c} 
& bid & ask \\
\hline$f_{u}>f_{d}$ & $1-\Psi^{\gamma}(1-p)$ & $\Psi^{\gamma}(p)$ \\
$f_{d}>f_{u}$ & $\Psi^{\gamma}(p)$ & $1-\Psi^{\gamma}(1-p)$ \\
\hline
\end{tabular}

Table 1: Distorted probabilities of upward move. 
$f_{d}=0$. We will employ the MINMAXVAR distortion with parameter $\gamma=0.25$. The riskneutral probability to move up is $p=0.4928$. Using the formulas in the above $f_{u}>f_{d}$ setting, we have a distorted probability to move up of $\left(1-\Psi^{\gamma}(1-p)\right)=0.3371$ to be used for the bid and $\Psi^{\gamma}(p)=0.6495$ to be used for the ask price calculation. This leads to the following prices:

$$
\begin{aligned}
\text { risk-neutral } A T M \text { call } & =\exp (-r h)\left(p f_{u}+(1-p) f_{d}\right)=2.9264 \\
\text { bid } A T M \text { call } & =\exp (-r h)\left(\left(1-\Psi^{\gamma}(1-p)\right) f_{u}+\Psi^{\gamma}(1-p) f_{d}\right)=2.0021 \\
\text { ask } A T M \text { call } & =\exp (-r h)\left(\Psi^{\gamma}(p) f_{u}+\left(1-\Psi^{\gamma}(p)\right) f_{d}\right)=3.8569
\end{aligned}
$$

For an ATM put, we have $f_{u}=0$ and $f_{d}=5.6100$. Since now $f_{d}>f_{u}$, we have a distorted probability to move up of $\Psi^{\gamma}(p)=0.6495$ to be used for the bid and $1-\Psi^{\gamma}(1-p)=0.3371$ to be used for the ask price calculation. This leads to the following prices:

$$
\begin{aligned}
\text { risk-neutral } A T M \text { put } & =\exp (-r h)\left(p f_{u}+(1-p) f_{d}\right)=2.8431 \\
\text { bid } A T M \text { put } & =\exp (-r h)\left(\Psi^{\gamma}(p) f_{u}+\left(1-\Psi^{\gamma}(p)\right) f_{d}\right)=1.9648 \\
\text { ask } A T M \text { put } & =\exp (-r h)\left(\left(1-\Psi^{\gamma}(1-p)\right) f_{u}+\Psi^{\gamma}(1-p) f_{d}\right)=3.7156
\end{aligned}
$$

It is worthwhile to note from this example that the base risk neutral price is generally not the mid-quote of the bid and ask price in a two price economy. In the first case the risk neutral price is below the mid-quote and in the second it is above.

\subsection{Conic Pricing Trinomial Trees}

In a trinomial tree, there is an additional middle state and the stock can now jump after a time-step of $h>0$ to move to three possible values, namely $u S_{0}, m S_{0}$ and $d S_{0}$, respresenting respectively the up-state, middle-state and down-state.

A common choice for the factors $u, m$ and $d$ are in terms of a given (yearly) volatility estimate $\sigma$ is

$$
\begin{aligned}
u & =\exp \left(\left(r-\sigma^{2} / 2\right) h+\sigma \sqrt{3 h}\right) \\
m & =\exp \left(\left(r-\sigma^{2} / 2\right) h\right) \\
d & =\exp \left(\left(r-\sigma^{2} / 2\right) h-\sigma \sqrt{3 h}\right)
\end{aligned}
$$

Consider now a derivative paying out $f_{u} \geq 0$ in the up-state, $f_{m} \geq 0$ in the middle-state and $f_{d} \geq 0$ in the downstate as in Figure 6 .

A possible set of risk-neutral probabilities are:

$$
p_{u}=1 / 6, \quad p_{m}=2 / 3 \quad \text { and } \quad p_{d}=1 / 6,
$$



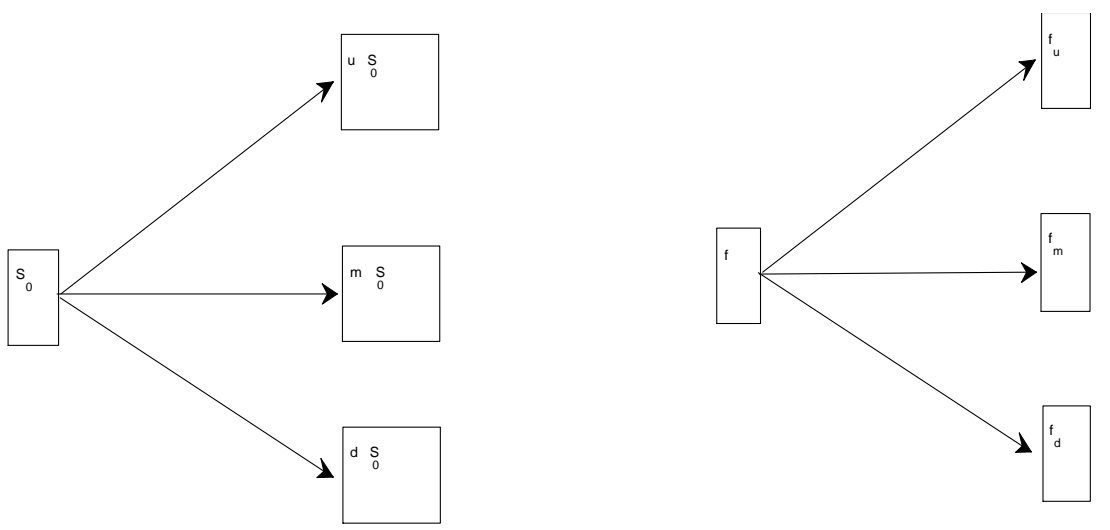

Figure 6: Trinomial tree

where $p_{u}, p_{m}$ and $p_{d}$ are respectively the risk-neutral probabilities to move to the up, middle or down state.

The current price $f$ of this derivative can be obtained in the risk-neutral setting as the discounted expected payoff under the risk-neutral measure.

$$
f=\exp (-r h)\left(p_{u} f_{u}+p_{m} f_{m}+p_{d} f_{d}\right)
$$

To calculate the bid price of this derivative, we have to first sort the possible payoffs $f_{u}, f_{m}$ and $f_{d}$. There are many possible combinations. We work out the case where $f_{m} \geq f_{d} \geq f_{u}$. Hence assume the lowest payoff is received in the up state and the highest payoff in the middle state.

For such a situation, the distribution functions are presented in Figure 7. The particular values used correspond to the setting of Example 11. We also show a distorted cdf. One observes that the distorted probability to receive $f_{u}$ is now $p_{u}^{*}=\Psi^{\gamma}\left(p_{u}\right)$. With a probability $p_{d}^{*}=\Psi^{\gamma}\left(p_{u}+p_{d}\right)-\Psi^{\gamma}\left(p_{u}\right)$ one gets $f_{d}$ and finally a payout $f_{m}$ is received under distortion with a probability $p_{m}^{*}=1-\Psi^{\gamma}\left(p_{u}+p_{d}\right)$. This leads to the bid price :

$$
\text { bid }=\exp (-r h)\left(p_{u}^{*} f_{u}+p_{m}^{*} f_{m}+p_{d}^{*} f_{d}\right) .
$$

Example 11 Assume a (yearly) volatility of $20 \%$ and a risk-free interest rate of $1 \%$ and $S_{0}=100$. Let us price under the above trinomial setting a derivative that pays out zero in the up case, one in the down state and three in the middle state:

$$
f_{u}=0, \quad f_{m}=3, \quad \text { and } \quad f_{d}=1 .
$$




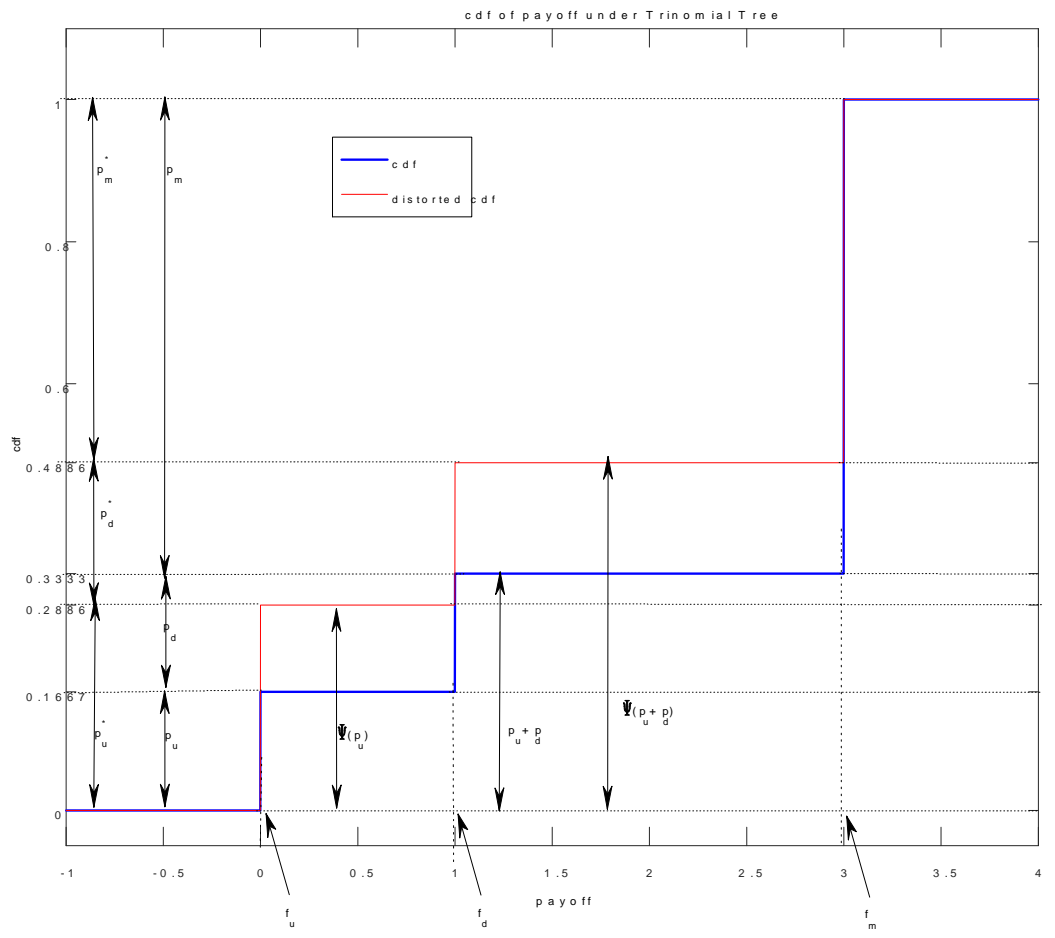

Figure 7: Trinomial distribution function and its distortion. 
With $\sigma=0.20$ and $h=1 / 12$, we have

$$
\begin{aligned}
u & =\exp \left(\left(r-\sigma^{2} / 2\right) h+\sigma \sqrt{3 h}\right)=1.1043 \\
m & =\exp \left(\left(r-\sigma^{2} / 2\right) h\right)=0.9992 \\
d & =\exp \left(\left(r-\sigma^{2} / 2\right) h-\sigma \sqrt{3 h}\right)=0.9041
\end{aligned}
$$

We will employ the MINMAXVAR distortion with parameter $\gamma=0.25$.

We start with the bid price calculation. The risk-neutral probability to move up is $p_{u}=$ 0.1667. We have a distorted probability to move up of $p_{u}^{*}=\Psi^{\gamma}\left(p_{u}\right)=0.2886$. Since $p_{u}+p_{d}=$ 0.3333 , we have that $\Psi^{\gamma}\left(p_{u}+p_{d}\right)=0.4886$ and hence that $p_{d}^{*}=\Psi^{\gamma}\left(p_{u}+p_{d}\right)-\Psi^{\gamma}\left(p_{u}\right)=0.2000$. Therefore, $p_{m}^{*}=1-\Psi^{\gamma}\left(p_{u}+p_{d}\right)=0.5114$.

For the ask price calculation we have to take the negative of the discounted distorted expectation of the negative payoff. Now $-f_{m} \leq-f_{d} \leq-f_{u}$, and hence we start with first considering the probability of a jump to the middle state $p_{m}=0.6666$. The distorted probability is now $\tilde{p}_{m}=\Psi^{\gamma}\left(p_{m}\right)=0.7990$. Further, we have that since $p_{m}+p_{d}=0.8333$, we have that $\Psi^{\gamma}\left(p_{m}+p_{d}\right)=0.9176$ and hence that $\tilde{p}_{d}=\Psi^{\gamma}\left(p_{m}+p_{d}\right)-\Psi^{\gamma}\left(p_{m}\right)=0.1186$. Therefore, $p_{u}^{*}=1-p_{m}^{*}-p_{d}^{*}=0.0824$.

This leads to the following prices:

$$
\begin{aligned}
\text { risk-neutral } & =\exp (-r h)\left(p_{u} f_{u}+p_{m} f_{m}+p_{d} f_{d}\right)=2.1649 \\
\text { bid } & =\exp (-r h)\left(p_{u}^{*} f_{u}+p_{m}^{*} f_{m}+p_{d}^{*} f_{d}\right)=1.7326 \\
\text { ask } & =\exp (-r h)\left(\tilde{p}_{u} f_{u}+\tilde{p}_{m} f_{m}+\tilde{p}_{d} f_{d}\right)=2.5136
\end{aligned}
$$

\subsection{Conic Hedging on Trees}

In this section, we elaborate on the conic hedging methodology as it applies to trees. We employ bid price and ask price functionals to construct hedging strategies. The primary focus of these strategies is to adopt positions maximizing bid prices or minimizing ask prices for the one step ahead risk being held or promised.

\subsubsection{Conic Delta Hedging on a Binomial and a Trinomial Tree}

In the context of trees we have considered different examples and calculated the risk-neutral, bid and their ask prices. Now we go, one step further. Instead of pricing just the derivative, we will now calculate the bid and ask prices of a package consisting of the derivative in combination with a stock position. More concretely, we consider the portfolio of 1 derivative and a position of $\alpha$ forward contracts paying out $\left(S_{T}-\exp (r T) S_{0}\right)$. Note that the risk-neutral upfront price to be paid for such a forward contract is zero and hence the risk-neutral price of the portfolio is unchanged. 


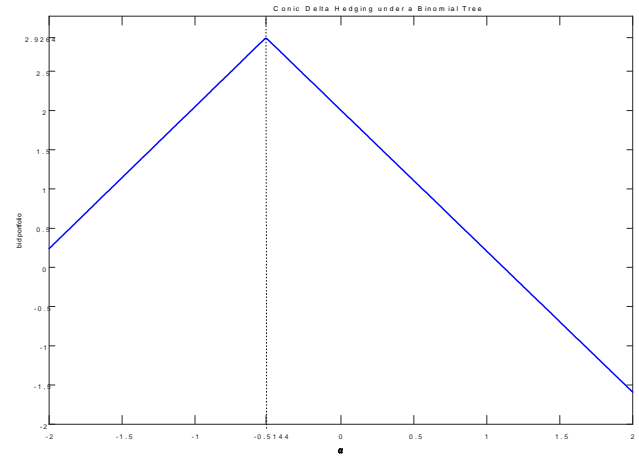

Figure 8: Delta hedging bid price as a function of the hedge position.

The key idea of conic hedging is that we look for an optimal value of $\alpha$. Optimal is here in the sense of having a maximal bid price or minimal ask price. Let us turn to our two concrete settings of the Binomial and Trinomial tree. Under the binomial tree the payoff in the up case now is $\pi_{u}=f_{u}+\alpha(u-\exp (r T)) S_{0}$, and it is $\pi_{d}=f_{d}+\alpha(d-\exp (r T)) S_{0}$ in the downstate. Assume for a given $\alpha$ that $\pi_{u} \geq \pi_{d}$, then we have that the bid price of this portfolio equals

$$
\text { bid }=\exp (-r h)\left(\left(1-\Psi^{\gamma}(1-p)\right) \pi_{u}+\Psi^{\gamma}(1-p) \pi_{d}\right) .
$$

In case, we would have for a given $\alpha$ that $\pi_{u} \leq \pi_{d}$, then we have that the bid price of this portfolio equals

$$
\text { bid } \left.=\exp (-r h)\left(\Psi^{\gamma}(p)\right) \pi_{u}+\left(1-\Psi^{\gamma}(p)\right) \pi_{d}\right) .
$$

Conic delta hedging looks for the $\alpha$, such that the bid price is maximal. We may observe in this context that the slope of the bid price function in the case $\pi_{u} \leq \pi_{d}$ is positive on account of $\Psi^{\gamma}(p)$ exceeding $p$ and noting that $p(u-\exp (r T))+(1-p)(d-\exp (r T))=0$ as forward is repriced under $p$. Similarly the slope in the case of $\pi_{u} \geq \pi_{d}$ is negative on account of $\Psi^{\gamma}(1-p)$ exceeding $1-p$. The maximum therefore occurs when the two expressions for the bid intersect and $\alpha$ is such that the two payoffs agree or

$$
\alpha^{*}=\frac{f_{u}-f_{d}}{(d-u) S_{0}}
$$

In Figure 8, we graph the bid price of the ATM call of Example 10 for different values of $\alpha$.

We see that the maximal value is attained for $\alpha^{*}=-0.5144$, the corresponding bid price equals for this $\alpha^{*}$ to 2.9264 , which is actually the risk-neutral price. This is actually no surprise, since $\alpha^{*}=-0.5144$ is also the traditional delta calculated under this setting and for this delta hedged position, the final portfolio payoff is risk-free and hence has the same 


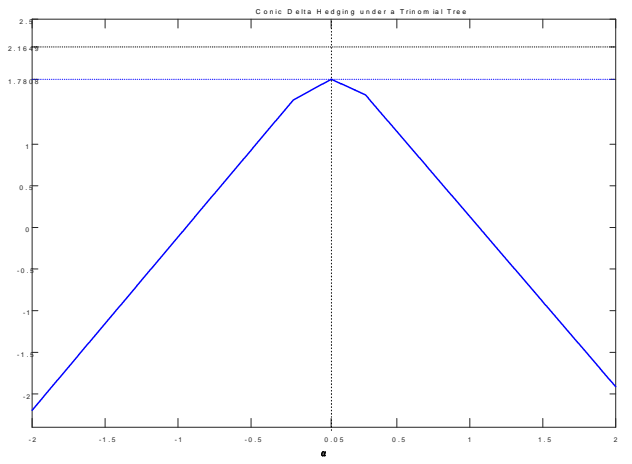

Figure 9: Delta hedging bid price as a function of the hedge position in the trinomial tree.

value in both the up-state and down-state. Our cdf hence is degenerate with just a jump of size 1 in this payoff. Distorting these cdf give rise to the same cdf, since there is nothing to distort. The bid price hence equals the risk-neutral price.

The situation is different though in the trinomial tree setting, which is an incomplete market setting. Now consider a derivative paying out $\pi_{u}=f_{u}+\alpha(u-\exp (r T)) S_{0}$ in the up-state, $\pi_{m}=f_{m}+\alpha(m-\exp (r T)) S_{0}$ in the middle-state and $\pi_{d}=f_{d}+\alpha(d-\exp (r T)) S_{0}$ in the downstate.

Using distorted expectations we can calculate the bid price of this portfolio and look for the $\alpha$, such that this bid price is maximized. In Figure 9, we graph the bid price of the setting of Example 11 for different values of $\alpha$.

We see that the maximal value is attained for $\alpha^{*}=0.05$, the corresponding bid price equals for this $\alpha^{*}$ to 1.7808 , which is below the risk-neutral price of 2.1649 and above the bid price 1.7326 of the unhedged derivative security.

A similar exercise can be made for the ask price and one can look for the $\alpha$ value for which the portfolio has the lowest ask price. We see, as in Figure 10 that the minimal ask price is attained for $\tilde{\alpha}=0.0500$, the corresponding ask price equals for this $\tilde{\alpha}$ to 2.4922 , which is above the risk-neutral price of 2.1649 and below the ask price 2.5136 for the unhedged derivative security.

We observe that the $\alpha^{*}$ giving rise to the maximal bid price equals $\tilde{\alpha}$ giving rise to the minimal ask price. This is a special feature of the trinomial model (and does not hold in general). In our situation and for this particular value, the portfolio's payoff in the up-state and down-state are exactly the same and conic delta hedging under a trinomial model brings us into a binomial model. 


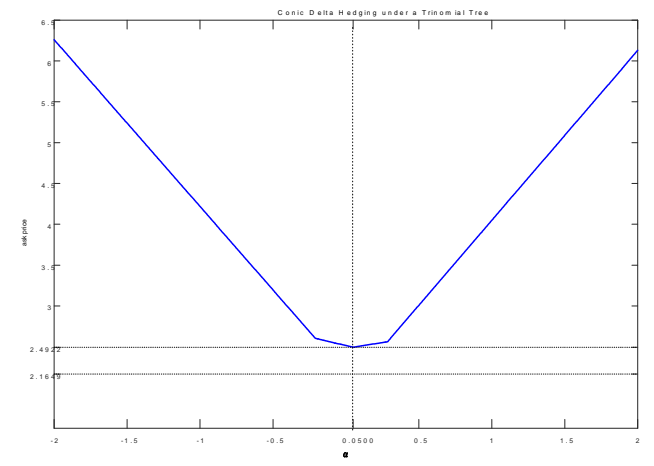

Figure 10: Delta hedging ask price as a function of the hedge position in the trinomial tree.

\subsubsection{Conic Delta Gamma Hedging on a Trinomial Tree}

One can expand the dynamic conic delta hedge by allowing for the possibility of investing in other instruments. One could use for example, allow for positions is a contract that pays at time $T$ a quadratic payoff. We call such a hedging conic delta-gamma hedging. As example, let us consider a swap contract that pays out at $T$ the square of the realized price change less the forward price for this claim:

$$
\left(S_{T}-S_{0} \exp (r T)\right)^{2}-E_{Q}\left[\left(S_{T}-S_{0} \exp (r T)\right)^{2}\right]
$$

Again, the upfront risk-neutral price is zero and hence a portfolio with our original derivative combined with $\alpha$ forward contracts paying out $\left(S_{T}-\exp (r T) S_{0}\right)$ and $\beta$ of the above quadratic contracts, has the same risk-neutral price as the derivative itself. However, the bid and the ask price of this package is not the same as just the bid and ask of the derivative. We can now perform a two-dimensional optimalization, looking for the optimal pairs $\left(\alpha^{*}, \beta^{*}\right)$ and $(\tilde{\alpha}, \tilde{\beta})$. Using distorted expectations we calculate the bid and ask prices of such a portfolio in the setting of example 11.

We obtain the maximal value for $\alpha^{*}=0.0334$ and $\beta^{*}=0.0248$. The corressponding portfolio's bid price equals 2.1649, which is the risk-neutral price. Similarly as in the case of the conic delta hedge in the Binomial model, we have that under the trinomial model, conic delta-gamma hedge eliminates all uncertainty in the payoff and therefore the portfolio's bid price equals the risk-neutral price.

\section{Multinomial Approximations}

Binomial approximations to the log normal model have been extensively employed in option pricing from their introduction in Cox, Ross and Rubinstein (1979). Extensions to trinomial 
models have been considered for example in Boyle (1988), Kamrad and Ritchken (1991). We now turn to the design of conic hedging strategies in a multinomial setting. In such an incomplete market setting risk may only be partially eliminated, leaving some residual risk. We proceed to construct multinomial approximations to Lévy processes.

We construct multinomial approximations to a Lévy process at unit time by splitting unit time into $N$ intervals of length $h=1 / N$. Suppose the characteristic function of the Lévy process $X=(X(t), 0 \leq t \leq 1)$ is given by $\phi(u)$ where

$$
\phi(u)=E[\exp (i u X(1))]
$$

Let $k(x)$ be the Lévy density for the Lévy process satisfying

$$
\phi(u)=\exp \left(\int_{-\infty}^{\infty}\left(e^{i u x}-1\right) k(x) d x\right)
$$

For the multinomial process we follow Yamada and Primbs (2004), Maller, Solomon and Szimayer (2006), and choose a step size $\Delta$ and $2 M$ jump sizes given by $\pm j \Delta$ for $j=1, \cdots, M$. The step size $\Delta$ will be determined later. For the jump size $x_{j}=-j \Delta, y_{j}=j \Delta$ we evaluate the arrival rates

$$
\begin{aligned}
A_{j} & =\int_{-\left(j+\frac{1}{2}\right) \Delta}^{-\left(j-\frac{1}{2}\right) \Delta} k(x) d x \\
B_{j} & =\int_{\left(j-\frac{1}{2}\right) \Delta}^{\left(j+\frac{1}{2}\right) \Delta} k(x) d x
\end{aligned}
$$

and define the conditional probabilities $p_{j}, q_{j}$ for a jump of size $x_{j}, y_{j}$ conditional on a jump by

$$
\begin{aligned}
p_{j} & =\frac{A_{j}}{\sum_{j}\left(A_{j}+B_{j}\right)} \\
q_{j} & =\frac{B_{j}}{\sum_{j}\left(A_{j}+B_{j}\right)}
\end{aligned}
$$

Let $p$ be the probability of no jump that is to be determined. The multinomial characteristic function is then $\phi_{M}(u ; p, \Delta)$ where

$$
\phi_{M}(u ; p, \Delta)=(1-p) \sum_{j=1}^{M}\left(p_{j} \exp \left(i u x_{j}\right)+q_{j} \exp \left(i u y_{j}\right)\right)+p
$$

Given $M$, and additionally fixing $N$ the multinomial characteristic function is determined 



Figure 11: Real and Imaginary Parts of the Theoretical and Multinomial Approximating Characteristic Functions. The multinomial is a 50 period 21 jump point process.

analytically from two parameters $p, \Delta$ the probability of no jump and the jump spacing. We determine $p, \Delta$ by a least squares minimization of

$$
z=\int_{-A}^{A}\left\|\phi(u)-\phi_{M}(u)^{N}\right\| d u
$$

for $M=10$ and $N=50$ with $X(1)$ given by a variance gamma process (Madan and Seneta (1990), Madan, Carr and Chang (1998)). The variance gamma process is obtained by time changing Brownian motion with drift $\theta$ and volatility $\sigma$ by a gamma process with unit mean rate and variance rate $\nu$. The parameter $\theta$ generates negative skewness when negative and typical risk neutral calibrations to the prices of options on the S\&P 500 index yield values ranging from -.1 to -.5 . Here we work, as an example, with the stylized annualized risk neutral parameters $\sigma=.2, \nu=.75$ and $\theta=-0.3$ and present in Figure 11 the theoretical and multinomial approximation in the range $|u| \leq 20$ to the real and imaginary parts of the characteristic function. We store one step ahead value processes on a non-uniform grid from which one may interpolate the one step ahead values needed.

\subsection{Dynamic Conic Delta and Gamma Hedging and other possi- bilities}

The various dynamic conic hedging strategies are presented in separate subsections. 


\subsection{Dynamic Conic Delta Hedging}

For the dynamic conic delta hedged claim the one step ahead random variable $X H(t+h)$ is defined by

$$
X H(t+h, a)=\left\{\begin{array}{cc}
V_{B}\left(S(t) e^{x_{i}}, t+h\right)+a S(t)\left(e^{x_{i}}-1\right) & \text { with probability } p_{i} \\
V_{B}(S(t), t+h) & \text { with probability } 1-\sum_{i} p_{i}-\sum_{j} q_{j} \\
V_{B}\left(S(t) e^{y_{j}}, t+h\right)+a S(t)\left(e^{y_{j}}-1\right) & \text { with probability } q_{j} .
\end{array}\right.
$$

and furthermore one has to optimize over the hedge position with a view to maximizing $V_{B H}(S(t), t)$ and minimizing the ask price when determining these prices. The hedge here involves just a position in the underlying stock. We then have

$$
\begin{aligned}
V_{B H}(S(t), t) & =\sup _{a} \int_{-\infty}^{\infty} x d \Psi\left(F_{X H(t+h, a)}(x)\right) \\
\delta_{B H}(S(t, t) & =\arg \sup _{a} \int_{-\infty}^{\infty} x d \Psi\left(F_{X H(t+h, a)}(x)\right) .
\end{aligned}
$$

The recursion (7) delivers the hedge bid prices along with the hedge deltas. One may then evaluate the benefits of dynamic delta hedging in two price economies working with a fixed distortion for the generation of market bid and ask prices. This program is implemented after developing multinomial approximations and selecting the distortion function defining market acceptability.

\subsection{Dynamic Conic Gamma Hedging}

On can expand the dynamic conic delta hedge in the definition of the one step ahead risk (6) by allowing for the possibility of investing in a short dated or weekly swap contract that pays out at the end of a week the realized squared price change less the forward price for this claim. Such moment based contracts have recently been introduced and studied in the context of moment risk premia in Kozhan, Neuberger and Schneider (2013). Assuming a risk neutral calibrated multinomial process we may define a dynamic conic (delta) gamma 
hedge as having the payout

$$
\begin{aligned}
X G H(t+h, a, b) & =\left\{\begin{array}{cc}
V_{B}\left(S(t) e^{x_{i}}, t+h\right)+a S(t)\left(e^{x_{i}}-1\right) & \text { with } \\
+b\left[S(t)^{2}\left(e^{x_{i}}-1\right)^{2}-C G\right] & \text { probability } p_{i} \\
V_{B}(S(t), t+h) & \text { with probability } \\
V_{B}\left(S(t) e^{y_{j}}, t+h\right)+a S(t)\left(e^{y_{j}}-1\right) & 1-\sum_{i} p_{i}-\sum_{j} q_{j} \\
+b\left[S(t)^{2}\left(e^{y_{j}}-1\right)^{2}-C G\right] & \text { with } \\
C G & =\sum_{i} p_{i} S(t)^{2}\left(e^{x_{i}}-1\right)^{2}+\sum_{j} q_{j} S(t)^{2}\left(e^{y_{j}}-1\right)^{2}
\end{array}\right.
\end{aligned}
$$

Importantly one may contrast the dynamic conic gamma hedge introduced here from the traditional approach of positioning to attain gamma neutrality in a number of ways. The traditional approach of gamma neutrality seeks to position in possibly long dated options to match the second derivative of the value function at the moment. We are concerned here with using options maturing at the next time step with a view to accessing a position in the future squared contract that helps us in raising or maximizing the current value, taken conservatively. The position in the squared contract is what we call the conic gamma hedge. This position is chosen in a different way as well. More precisely, we maximize current bid prices and do not match current derivatives. Hence we emphasize that dynamic conic gamma hedging as defined here is fundamentally different from gamma neutrality and in fact our portfolios are typically neither delta nor gamma neutral.

The bid price is then defined by

$$
\begin{aligned}
V_{B G H}(S(t), t) & =\sup _{a, b} \int_{-\infty}^{\infty} x d \Psi\left(F_{X G H(t+h, a, b)}(x)\right) \\
{\left[\delta_{B G H}\left(S(t, t), \gamma_{B G H}(S(t), t)\right]\right.} & =\arg \sup _{a, b} \int_{-\infty}^{\infty} x d \Psi\left(F_{X G H(t+h, a, b)}(x)\right) .
\end{aligned}
$$

The recursions of equations (10)-(11) deliver dynamic conic delta and gamma hedged competitive bid prices. Ask prices are analogously obtained by taking the negative of the bid price for the negative. The weekly gamma hedge can now be actually implemented by the use of weekly options for which there is an active market for numerous underliers. The hedge for the square just being the purchase of all out of the money puts and calls. Other hedges may be similarly defined with details available in a longer version of the paper. 


\section{Results on Dynamic Conic Hedging For Competi- tiveness}

We report on the hedging results for two contracts in two subsections. The first is a one year 90, 110 strangle with payoff

$$
V_{S}(1)=\max (S(1)-110,0)+\max (90-S(1), 0) .
$$

The second is a capped strangle with payoff

$V_{C S}(1)=\max (S(1)-110,0)+\max (90-S(1), 0)-\max (S(1)-120,0)-\max (80-S(1), 0)$.

For the strangle we report on a number of parameterizations for an underlying variance gamma Lévy process approximated by a 21 point multinomial. This helps in obtaining an understanding of the effects of various parametric effects on the associated dynamic delta hedge. The base parameterization is that for which we have reported on in Section 3. This parameterization is used for dynamic gamma hedging. For the capped strangle we report on just the base parameterization and the Gaussian special case. Joint dynamic gamma and skew hedging is implemented for just the most complicated product of the capped strangle reflecting sign changes in both the delta and the gamma. The hedging time step in all cases is $1 / 50$ or approximately one week. As already mentioned weekly options are available for the operational implementation of such hedges. Results are presented graphically and in short summary tables. The graph at the first date is furthest from maturity and this is the graph mainly presented. Occasionally we present graphs for intermediate dates. The results are all stored on a 800 point non-uniform space grid for 50 time steps in numerous $800 \times 50$ matrices for the bid and ask values and the associated positions $a, b, c$ as the case may be. The stress levels for local acceptability are $\gamma=.01+.25 h$ except in the high stress cases reported where it is $.05+.75 h$. When we implement dynamic skew hedging in addition to gamma hedging the multinomial approximation is taken to be with 51 points, 25 up and down and the no change point.

\subsection{Results on the strangle}

We begin the reporting with the unhedged and delta hedged bid and ask prices for the base parameterization.

\subsection{The unhedged and delta hedged bid and ask values}

Figure 12 presents the time one bid and ask prices for the base case with and without the hedge. 


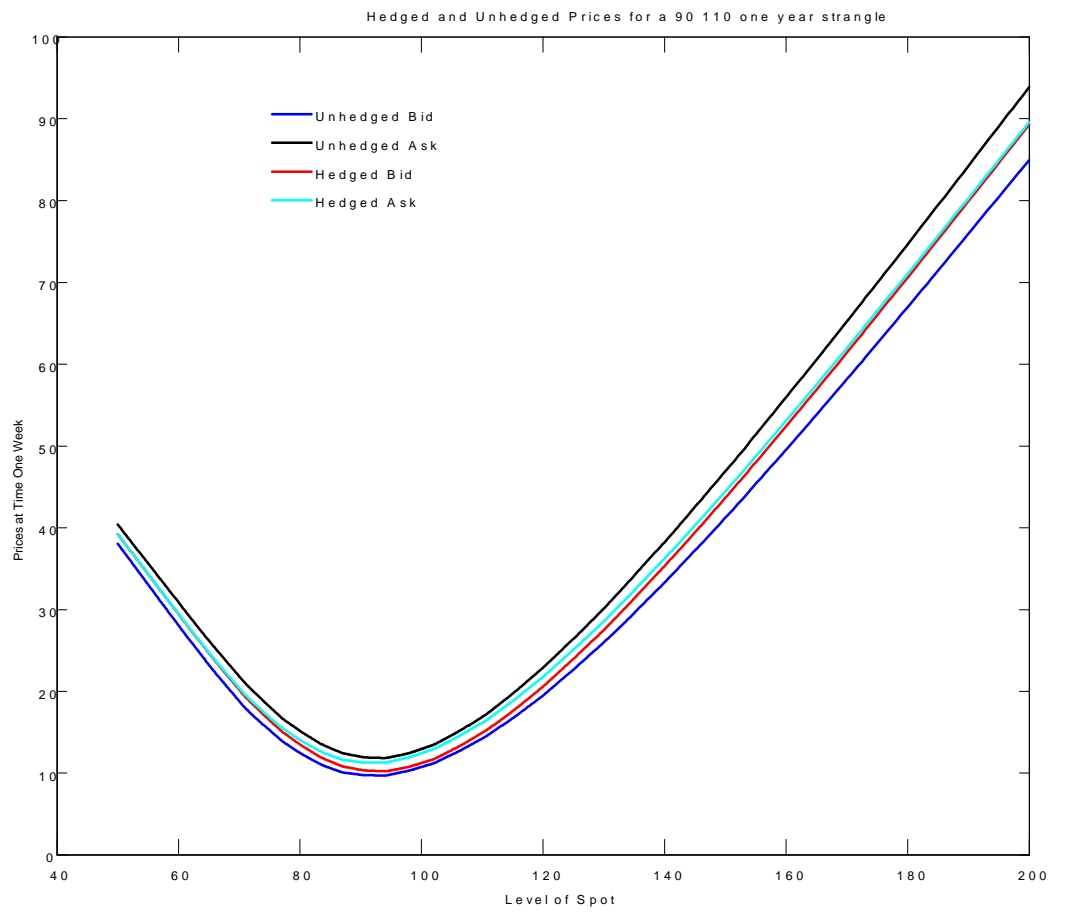

Figure 12: Strangle one year unhedged and hedge bid and ask prices as function of the spot at unit time. 
The unhedged ATM spread is around $20 \%$ and is reduced to $10 \%$ with the dynamic delta hedge that maximizes the bid price and minimizes the ask price. We present in Table 1 the bid and ask prices, hedged and unhedged at times 1, 25 and 37 for the spot at 85, 100 and 115 .

\begin{tabular}{llllll}
\multicolumn{2}{c}{ Table 1} & & & & \\
& Spot & Bid & Bid & Ask & Ask \\
Time & Price & Unhedged & Hedged & Hedged & Unhedged \\
& 85 & 10.61 & 11.42 & 12.16 & 13.04 \\
1 & 100 & 10.74 & 11.22 & 12.41 & 12.93 \\
& 115 & 16.70 & 17.56 & 18.79 & 19.69 \\
& 85 & 7.59 & 8.13 & 8.48 & 9.06 \\
25 & 100 & 5.52 & 5.68 & 6.37 & 6.55 \\
& 115 & 12.36 & 12.69 & 13.53 & 13.86 \\
37 & 85 & 5.50 & 5.86 & 6.01 & 6.39 \\
& 100 & 2.51 & 2.59 & 2.95 & 3.04 \\
& 115 & 9.57 & 9.69 & 10.20 & 10.32
\end{tabular}

\subsubsection{Comparison of Deltas with Value Function Derivatives}

We may observe that the deltas or hedge positions taken lie above the negative of the derivative in this left skewed market. We basically buy more than the derivative when we buy to further protect against the considerably more down moves that are possible in the base measure. To confirm such a conjecture we ran an experiment with a positive skew where the value of $\theta$ was switched to 0.3 . For a positive skew we confirmed that the value optimizing deltas or hedge positions fall below the negative of the derivative.

\subsection{Results on the capped strangle}

The results reported on here are for the base case for both the stochastic processes and the stress levels. The only difference is that for the joint gamma and skew hedged case we used a 51 point multinomial approximation.

\subsection{The unhedged and delta hedged values}

We begin with the delta hedged and unhedged bid and ask prices for the capped strangle. Figure 13 presents these set of prices.

This is a more complex contract with considerable variations in its gamma structure and a simple delta hedge reduces the spread but not by that much. Table 2 presents the values 


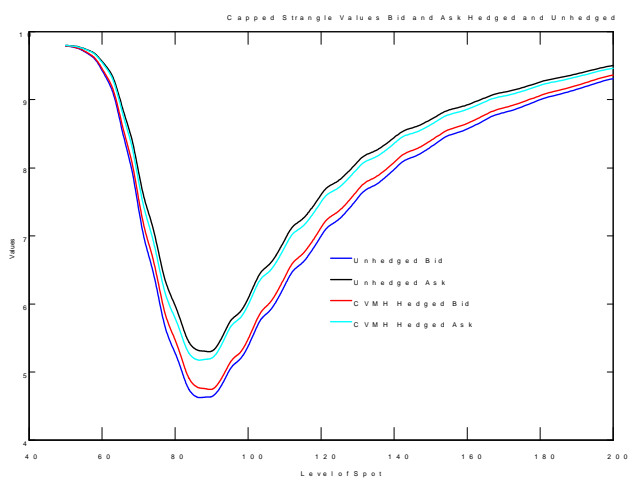

Figure 13: Bid and Ask Values Delta hedged and unhedged for the capped strangle.

at the indicated times and spot levels.

Table 2

Spot Bid Bid Ask Ask

Time Price Unhedged Hedged Hedged Unhedged

$\begin{array}{lllll}85 & 4.66 & 4.82 & 5.20 & 5.36\end{array}$

$\begin{array}{llllll}1 & 100 & 5.38 & 5.48 & 5.98 & 6.08\end{array}$

$\begin{array}{lllll}115 & 6.60 & 6.73 & 7.13 & 7.24\end{array}$

$\begin{array}{lllll}85 & 3.82 & 3.96 & 4.20 & 4.34\end{array}$

$\begin{array}{llllll}25 & 100 & 3.39 & 3.43 & 3.81 & 3.85\end{array}$

$\begin{array}{lllll}115 & 6.74 & 6.81 & 7.18 & 7.24\end{array}$

$\begin{array}{lllll}85 & 3.11 & 3.20 & 3.41 & 3.50\end{array}$

$\begin{array}{llllll}37 & 100 & 1.49 & 1.51 & 1.72 & 1.75\end{array}$

$\begin{array}{lllll}115 & 6.94 & 6.98 & 7.28 & 7.31\end{array}$

\subsection{Comparison of Alternative Hedge Designs}

We next investigate the further competitive benefits arising from the use of different hedging instruments. In this regard we compare gamma hedging the capped strangle with positions in the gamma contract, or the straddle contract, and then consider joint hedging with positions in both the gamma and the skew contract and joint hedging with positions in $5 \%$ out of the money puts and calls. Table 3 presents these results. For the spot at 100 the capped strangles are better hedged by a gamma hedge as opposed to a straddle hedge. The joint gamma skew hedge improves the spread somewhat. The most competitive hedging instruments in this collection turn out to be positioning in $5 \%$ out of the money puts and calls that allow for 
some asymmetric positioning on the two sides of at the money.

Table 3

$\begin{array}{llllllllll} & & \text { Bid } & \text { Bid } & \text { Bid } & \text { Bid } & \text { Ask } & \text { Ask } & \text { Ask } & \text { Bid } \\ \text { Time } & \text { Spot } & \text { Straddle } & \text { Gamma } & \text { Gamma } & \text { Put } & \text { Put } & \text { Gamma } & \text { Gamma } & \text { Straddle } \\ \text { Time } & \text { Price } & \text { Hedge } & \text { Hedge } & \text { Skew } & \text { Call } & \text { Call } & \text { Skew } & \text { Hedge } & \text { Hedge } \\ & 85 & 4.88 & 4.87 & 4.92 & 4.94 & 5.07 & 5.09 & 5.15 & 5.13 \\ 1 & 100 & 5.53 & 5.57 & 5.61 & 5.62 & 5.84 & 5.86 & 5.89 & 5.94 \\ & 115 & 6.76 & 6.77 & 6.82 & 6.81 & 7.05 & 7.04 & 7.08 & 7.10 \\ & 85 & 3.99 & 3.98 & 4.02 & 4.02 & 4.14 & 4.14 & 4.18 & 4.17 \\ 25 & 100 & 3.48 & 3.51 & 3.54 & 3.55 & 3.67 & 3.69 & 3.72 & 3.75 \\ & 115 & 6.83 & 6.84 & 6.87 & 6.87 & 7.11 & 7.11 & 7.14 & 7.15 \\ 37 & 85 & 3.22 & 3.22 & 3.24 & 3.26 & 3.34 & 3.37 & 3.38 & 3.39 \\ & 100 & 1.55 & 1.54 & 1.57 & 1.57 & 1.65 & 1.66 & 1.68 & 1.68 \\ & 115 & 6.99 & 7.01 & 7.04 & 7.07 & 7.19 & 7.21 & 7.23 & 7.26\end{array}$

\section{Conclusion}

The paper provides a new hedging methodology, a quantitative analysis of firm value and hedge interaction, permitting systematic hedging choices that have a wide range of applications. More precisely dynamic sequences of bid and ask prices are constructed by locally evaluating the one step ahead risk as a distorted expectation to produce conservative valuation operators as examples of nonlinear expectations. The pricing operators are employed to illustrate the competitiveness of hedging strategies as reflected by improving post hedge bid and ask prices. The hedging instruments are expanded from positions in the stock to also include access to squared, absolute and cubed returns termed respectively as dynamic gamma, skew and straddle hedging. Also investigated are the use of $5 \%$ out of the money put and call options. The hedge and valuation procedures are illustrated for a variety of processes approximated by a multinomial evolution. It is observed that for the lower stress levels in distortion dynamic delta and gamma hedging can induce replication and a convergence of the bid and ask prices. More generally one observes the advantage of gamma hedging over straddle hedging and the advantage of adding dynamic skew hedges to the hedge design. The most competitive hedge design for strangles among the ones reported on are positioning in $5 \%$ out of the money puts and call options. The procedures presented make available an analysis of the relative quality of different hedge instruments, for different products in varying underlying stochastic risk structures. 


\section{References}

[1] Adam, T. R. and C. R. Fernando (2006), "Hedging, Speculation and Shareholder Value," Journal of Financial Economics, 81, 283-309.

[2] Allayannis, G. and A. P. Weston (2001), "The Use of Foreign Currency Derivatives and Firm Market Value," Review of Financial Studies, 14, 243-276.

[3] Barrieu, P., and N. El Karoui (2005), "Inf convolution of risk measures and optimal risk transfer," Finance and Stochastics, 9, 269-298.

[4] Bartram, S. M., G. W. Brown and J. Conrad (2011), "The Effects of Derivatives on Firm Risk and Value," Journal of Financial and Quantitative Analysis, 46, 967-999.

[5] Basak, S. and G. Chabakauri (2012), "Dynamic Hedging in Incomplete Markets; A Simple Solution," Review of Financial Studies, 25, 1845-1896.

[6] Bertsimas, D., L. Kogan and A. Lo (2001), "Hedging Derivative Securities and Incomplete Markets: An $\varepsilon$ Arbitrage Approach," Operations Research, 49, 372-397.

[7] Bion-Nadal, J. (2008), "Dynamic Risk Measures: Time Consistency and Risk Measures from BMO martingales," Finance and Stochastics, 12, 219-244.

[8] Bion-Nadal, J. (2009), "Time Consistent Dynamic Risk Processes," Stochastic Processes and their Applications, 119, 633-654.

[9] Black, F. and M. Scholes, 1973, "The Pricing of Options and Corporate Liabilities," Journal of Political Economy, 81, 637-654.

[10] Boyle, P. (1988), "A Lattice Framework for Option Pricing with Two State Variables," Journal of Financial and Quantitative Analysis, 23, 1-12.

[11] Campello, M., C. Lin, Y. Ma and H. Zou (2011), "The Real and Financial Implications of Corporate Hedging," Journal of Finance, 66, 1615-1647.

[12] Carr, P., "Semi-Static Hedging of Barrier Options under Poisson Jumps," International Journal of Theoretical and Applied Finance, 14, 1091-1111.

[13] Carr, P., K. Ellis and V. Gupta (1998), "Static Hedging of Exotic Options," Journal of Finance, 53, 1165-1190.

[14] Carr, P., H. Geman and D. Madan (2001), "Pricing and Hedging in Incomplete Markets," Journal of Financial Economics, 62, 131-167. 
[15] Carr, P., D. B. Madan and J. J. Vicente-Alvarez (2011), "Markets, Profits, Capital, Leverage and Returns," Journal of Risk, 14, 95-122.

[16] Carr, P., and L. Wu (2014), "Static Hedging of Standard Options," Journal of Financial Econometrics, 12, 3-46.

[17] Carmona, R. (2009), Ed. Indifference Pricing, Theory and Applications, Princeton University Press, Princeton, NJ.

[18] Chateauneuf, A. (1991), "On the use of capacities in modeling uncertainty averaion and risk aversion," Journal of Mathematical Economics, 20, 343-369.

[19] Cheridito, P., F. Delbaen and M. Kupper (2006), "Dynamic monetary risk measures for bounded discrete time processes," Electronic Journal of Probability, 11, 57-106.

[20] Chen, S., C. Lee and K. Shrestha (2001), "On the mean-generalized semivariance approach to determining the hedge ratio," The Journal of Futures Markets, 21, 581-598.

[21] Cherny, A. and D. Madan (2009), "New Measures for Performance Evaluation," Review of Financial Studies, 22, 2571-2606.

[22] Cherny, A. and D. B. Madan (2010), "Markets as a Counterparty: An Introduction to Conic Finance," International Journal of Theoretical and Applied Finance, 13, 11491177.

[23] Christoffersen, P., R. Elkhami, B. Feunou and K. Jacobs (2010), "Option Valuation with Conditional Heteroskedasticity and Non-Normality," Review of Financial Studies, 23, 2139-2183.

[24] Cochrane, J. H. (2001), Asset Pricing, Princeton University Press, Princeton, NJ.

[25] Cochrane, J. H. and J. Saá-Requejo (2000), "Beyond Arbitrage: 'Good Deal' Asset Price Bounds in Incomplete Markets," Journal of Political Economy, 108, 79-119.

[26] Cohen, S., and R. J. Elliott, (2010), "A General Theory of Finite State Backward Stochastic Difference Equations," Stochastic Processes and their Applications 120, 442466.

[27] Cox, J. C., S. A. Ross and M. Rubinstein (1979), "Option Pricing: A Simplified Approach," Journal of Financial Economics, 7, 229-263.

[28] Cvitanić, J. (2000), "Minimizing expected loss of hedgin in incomplete and constrained markets," SIAM Journal of Control and Optimization, 38, 1050-1066. 
[29] Cvitanić, J. and I. Karatazas (1996), "Hedging and Portfolio Optimization under Transactions Costs: A Martingale Approach," Mathematical Finance, 6, 133-165.

[30] Cvitanić, J. and F. Zapatero (2004), Introduction to the Economics and Mathematics of Financial Markets, First Edition, The MIT Press.

[31] Delbaen, F. (2000), "Coherent Risk Measures on General Probability Spaces," Department of Mathematics, ETH, Zurich,

[32] Detlefsen, K., and G. Scandolo (2005), "Conditional and dynamic convex risk measures," Finance and Stochastics, 9, 539-561.

[33] Eberlein, E. and D. B. Madan (2009), "Hedge Fund Performance: Sources and Measures," International Journal of Theoretical and Applied Finance, 12, 267-282.

[34] Eberlein, E. and D. B. Madan (2011), "Unbounded liabilities, capital reserve requirements and the taxpayer put option," Quantitative Finance, 12, 709-724.

[35] Eberlein, E., D. B. Madan, M. Pistorius, W. Schoutens and M. Yor (2012), "Two Price Economies in Continuous Time," Annals of Finance, DOI 10.1007/s10436-013-0228-3.

[36] Eberlein, E., D. B. Madan, M. Pistorius, and M. Yor (2014), "Bid and Ask Prices as Non-Linear Continuous Time G-Expectations Based on Distortions ," Mathematics and Financial Economics, forthcoming.

[37] Föllmer, H. and P. Leukert (2000), "Efficient Hedging: Cost versus shortfall risk," Finance and Stochastics, 4, 117-146.

[38] Föllmer, H. and I. Penner (2006), "Convex Risk Measures and the Dynamics of Penalty Functions," Statistics and Decisions, 24, 61-96.

[39] Föllmer, H. and A. Schied (2004), Stochastic Finance: An Introduction in Discrete Time, second edition, de Gruyter studies in mathematics, 27.

[40] Galichon, A. and M. Henry (2012), "Dual theory of choice under multivariate risks," Journal of Economic Theory, 147, 1501-1516.

[41] Gilboa, I. and D. Schmeidler (1989), "Maxmin expected utility with non-unique prior," Journal of Mathematical Finance, 18, 141-153.

[42] Hansen, L. and T. Sargent (2001), "Robust Control and model uncertainty," American Economic Review, 91, 60-66.

[43] Hansen, L. and T. Sargent (2007), Robustness, Princeton University Press, Princeton, New Jersey. 
[44] Heath, D., E. Platen and M. Schweizer (2001), "A Comparison of Two Quadratic Approaches to Hedging in Incomplete Markets," Mathematical Finance, 11, 385-413.

[45] International Capital Markets Association (2012), "Haircuts and Initial Margins in the Repo Market," European Repo Council Report, Compiled by Richard Comotto.

[46] Iyengar, G. and A. Ma (2010), "A robust optimization approach to pension fund management," Journal of Asset Management, 11, 163-177.

[47] Jin, Y. and P. Jorion (2006), "Firm Value and Hedging: Evidence from the U.S. Oil and Gas Producers." Journal of Finance 61, pp. 893-919.

[48] Jin, H. Z. Xu and X. Y. Zhou (2008), "Behavioral Portfolio Selection in Continuous Time," Mathematical Finance, 18, 385-426.

[49] Jobert, A. and L. C. G. Rogers (2008), "Valuations and Dynamic Convex Risk Measures," Mathematical Finance, 18, 1-22.

[50] Jouini, E. and H. Kallal (1995), "Martingales and Arbitrage in Securities Markets with Transactions Costs," Journal of Economic Theory, 66, 178-197.

[51] Kabanov, Y. (1999), "Hedging and Liquidation under Transactions Costs in Currency Markets," Finance and Stochastics, 3, 237-248.

[52] Kamrad, B. and P. Ritchken (1991), "Multinomial Approximating Models for Options with $k$ State Variables," Management Science, 37, 1640-1652.

[53] Klibanoff, P., M. Marrinacci and S. Mukerji (2005), "A smooth model of decision making under ambiguity," Econometrica, 73, 1849-1892.

[54] Kolkiewicz, A. and Y. Liu (2012), "Semi-Static Hedging for GMWB in Variable Annutities," North American Actuarial Journal, 16, 112-140.

[55] Kozhan, R., A. Neuberger and P. Schneider (2013), "The Skew Risk premium in the Equity Index Market," Review of Financial Studies, doi 10.1093/rfs/hht039.

[56] Kusuoka, S. (2001), "On Law Invariant Coherent Risk Measures," Advances in Mathematical Economics, 3, 83-95.

[57] Madan, D. B. (2014a), "Asset Pricing Theory for Two Price Economies," Annals of Finance, DOI 10.1007/s10436-014-0255-8.

[58] Madan, D. B. (2014b), "Conic Portfolio Theory," Working Paper, Robert H. Smith School of Business, University of Maryland. 
[59] Madan, D., P. Carr and E. Chang (1998), "The Variance Gamma Process and Option Pricing," European Finance Review, 2, 79-105.

[60] Madan, D., M. Pistorius and M. Stadje (2015a), "Convergence of BSDeltaE's Driven by Random Walks to BSDE's: The case of (in) Finite Activity Jumps with General Driver," arXiv: 1406.7145 [math.PR].

[61] Madan, D., M. Pistorius and M. Stadje (2015b), "On Dynamic Spectral Risk Measures and a Limit Theorem," arXiv:1301.3531 [math.PR].

[62] Madan, D.B. and W. Schoutens (2012), "Tenor Specific Pricing," International Journal of Theoretical and Applied Finance, 15, 6, DOI:10.1142/S0219024912500434.

[63] Madan, D. B. and W. Schoutens (2011). "Conic Finance and the Corporate Balance Sheet," International Journal of Theoretical and Applied Finance, 14, 587-610.

[64] Madan, D. and E. Seneta (1990), "The Variance Gamma (V.G.) Model for Share Market Returns," Journal of Business, 63, 511-524.

[65] Madan, D. B. and Y. Sharaiha (2014), "Option Overlay Strategies," Working Paper, Robert H. Smith School of Business.

[66] Maenhout, P. (2004), "Robust portfolio rules and asset pricing," Review of Financial Studies, 17, 951-983.

[67] Maller, R., D. H. Solomon and A. Szimayer (2006), "A Multinomial Approximation for American Option Prices in Lévy Process Models," Mathematical Finance, 16, 613-633.

[68] Markowitz, H. M. (1952), "Portfolio Selection," Journal of Finance, 7, 77-91.

[69] Marshall, C., M. Hardy and D. Saunders (2014), "Measuring the effectiveness of static hedging strategies for a guaranteed minimum income benefit," North American Actuarial Journal, doi 10.1080/10920277.2012.10590636.

[70] Merton, R. C. (1971), "Optimum Portfolio and Consumption Rules in a ContinuousTime Model," Journal of Economic Theory, 3, 371-413.

[71] Merton, R., 1973, "Theory of Rational Option Pricing", Bell Journal of Economics and Management Science, 4, 141-183.

[72] Modigliani, F., and M. Miller (1958), "The Cost of Capital, Corporation Finance and the Theory of Investment," American Economic Review, 48, 261-297.

[73] Musiela, M. and T. Zariphopoulou (2004), " A Valuation Algorithm for Indifference Prices in Incomplete Markets," Finance and Stochastics, 8, 399-414. 
[74] Øksendahl, B. and A. Sulem (2009), "Maximum Principles for Optimal Control of Forward-Backward Stochastic Differential Equations with Jumps," SIAM Journal of Optimization and Control, 48, 2945-2976.

[75] Phillips, P. M. (2013), "Simulation and Testing of Semi-Static Hedging Solutions for Investment Products with Guarantees," Equity Based Insurance Guarantees Conference, November 18-19, Atlanta GA.

[76] Ross, S. A. (1978), "A Simple Approach to the Valuation of Risky Streams," Journal of Business, 51, 453-475.

[77] Schachermayer, W. (2004), "The Fundamental Theorem of Asset Pricing under Proportional Transactions Costs in Finite Discrete Time," Mathematical Finance, 14, 19-48.

[78] Schmeidler, D. (1986), "Integration Representation without Additivity," Proceedings of the American Mathematical Society, 97, 255-261.

[79] Sekine, J. (2004), "Dynamic Minimization of Worst Conditional Expectation of Shortfall," Mathematical Finance, 14, 605-618.

[80] Shen, S. A. Pelsser and P. Schotman (2013), "Robust Hedging in Incomplete Markets," Working Paper, Maastricht University.

[81] Staum, J. (2004), "Fundamental theorems for asset pricing for good deal bounds," Mathematical Finance, 14, 141-161.

[82] Staum, J. (2008), "Incomplete Markets," In Handbooks in OR $\mathscr{E} M S S$, 15, Eds. J. R. Birge and V. Linetsky, Elsevier. DOI 10.1016/S0927-0507(07)15012-X.

[83] Yamada, Y. and J. Primbs (2004), "Properties of multinomial lattices with cumulants for option pricing and hedging," Asia Pacific Financial Markets, 11, 335-365.

[84] Yaari, M. (1987), "The Dual Theory of Choice under Risk," Econometrica, 55, 95-115.

[85] Yaari, M. (1986), "Univariate and multivariate comparisons of risk aversion: a new approach," in: W. Heller, R. Starr, D. Starett (Eds.), Essays in Honor of Kenneth Arrow, Cambridge University Press, 173-187. 